\title{
Auxin Profiling and GmPIN Expression in Phytophthora sojae-Soybean Root Interactions
}

\author{
Anna K. Stasko,, ${ }^{1,2}$ Amine Batnini,,1,2 Carlos Bolanos-Carriel,1,2 Jinshan Ella Lin,, ${ }^{3}$ Yun Lin, ${ }^{3}$ Joshua J. Blakeslee,,3,4 and \\ Anne E. Dorrance $1,2,4, \dagger$ \\ ${ }^{1}$ Department of Plant Pathology, The Ohio State University, Wooster, OH 44691 \\ ${ }^{2}$ Center for Soybean Research, The Ohio State University, Wooster, OH 44691 \\ ${ }^{3}$ Department of Horticulture and Crop Science and OARDC Metabolite Analysis Cluster, The Ohio State University, Wooster, OH 44691 \\ ${ }^{4}$ Center for Applied Plant Sciences, The Ohio State University, Columbus, OH 43210 \\ Accepted for publication 23 June 2020.
}

\begin{abstract}
Auxin (indole-3-acetic acid, IAA) has been implicated as a susceptibility factor in both beneficial and pathogenic molecular plant-microbe interactions. Previous studies have identified a large number of auxin-related genes underlying quantitative disease resistance loci (QDRLs) for Phytophthora sojae. Thus, we hypothesized that auxin may be involved the $P$. sojae-soybean interaction. The levels of IAA and related metabolites were measured in mycelia and media supernatant as well as in mock and inoculated soybean roots in a time course assay. The expression of 11 soybean Pin-formed (GmPIN) auxin efflux transporter genes was also examined. Tryptophan, an auxin precursor, was detected in the $P$. sojae mycelia and media supernatant. During colonization of roots, levels of IAA and related metabolites were significantly higher in both moderately resistant Conrad and moderately susceptible Sloan inoculated roots compared with mock controls at $48 \mathrm{~h}$ postinoculation
\end{abstract}

ABSTRACT (hpi) in one experiment and at $72 \mathrm{hpi}$ in a second, with Sloan accumulating higher levels of the auxin catabolite IAA-Ala than Conrad. Additionally, one GmPIN at $24 \mathrm{hpi}$, one at $48 \mathrm{hpi}$, and three at $72 \mathrm{hpi} \mathrm{had}$ higher expression in inoculated compared with the mock control roots in Conrad. The ability of resistant cultivars to cope with auxin accumulation may play an important role in quantitative disease resistance. Levels of jasmonic acid (JA), another plant hormone associated with defense responses, were also higher in inoculated roots at these same time points, suggesting that JA also plays a role during the later stages of infection.

Keywords: auxin, biochemistry and cell biology, genetics and resistance, metabolite profiling, Phytophthora, PIN genes, quantitative disease resistance, roots, soybean.
Although the phytohormone auxin is best known for playing various roles in plant growth and development as well as responses to environmental stimuli (reviewed in Benjamins and Scheres 2008; Friml et al. 2002a, 2002b; Gao et al. 2014; Rightmyer and Long 2011; Rosendahl and Jochimsen 1995; Roy et al. 2017; reviewed in Woodward and Bartel 2005), several recent studies suggest that it may also play a role in plant-microbe interactions. In this context, it often enhances susceptibility to ensure establishment of a

†Corresponding author: A. E. Dorrance; dorrance.1@osu.edu

Current address for A. K. Stasko: Department of Plant Pathology, North Dakota State University, Fargo, ND 58108, U.S.A.

Current address for J. E. Lin: Nutrient and Phytochemical Analytics Shared Resource, The Ohio State University, Columbus, OH 43210, U.S.A.

Current address for Y. Lin: By-Health Co., Ltd., Zhuhai, 519040, People's Republic of China.

Funding: This project was funded by the United Soybean Board (USB) projects 1402-532-5659, 1720-172-0125, and 1920-172-0110. Salaries and research support for this project were provided by State and Federal Funds appropriated to the Ohio Agricultural Research and Development Center, the Ohio State University, the Ohio State University Centers for Applied Plant Sciences and for Soybean Research, and the National Institute of Food and Agriculture, U.S. Department of Agriculture, Hatch projects $\mathrm{OHO} 01303$ and $\mathrm{OHO} 01426$. This project was also funded through check off dollars provided by the Ohio Soybean Council. A. K. Stasko was supported by the American Society of Agronomy USB Fellowship. Additional support was provided by the Ohio State University Center for Applied Plant Sciences as part of the Soybean Resistance Team project.

*The $\boldsymbol{e}$-Xtra logo stands for "electronic extra" and indicates that one supplementary figure and one supplementary table are published online.

The author(s) declare no conflict of interest.

(c) 2020 The American Phytopathological Society mutualistic or pathogenic microbe in plant tissues (Berens et al. 2017; Boivin et al. 2016; Kunkel and Harper 2018; Ludwig-Müller 2015; Ma and Ma 2016; Robert-Seilaniantz et al. 2011; Spaepen et al. 2007). The most widely known example of this is Agrobacterium tumefaciens, which induces auxin biosynthesis through the introduction of auxin biosynthesis genes into the host genome (Chilton et al. 1977; Thomashow et al. 1984). Several pathogens, including numerous Pseudomonas syringae and Pseudomonas savastanoi pathovars (Glickmann et al. 1998; McClerklin et al. 2018), the fungal pathogen Taphrina deformans (Perley and Stowe 1966), and a wheat rust pathogen, Puccinia graminis f. sp. tritici (Yin et al. 2014), produce indole-3-acetic acid (IAA), the primary auxin in plants (Korasick et al. 2013; Tivendale et al. 2014). This hormone contributes to gall formation in host tissue and is required for pathogenicity by Pseudomonas savastanoi (Glickmann et al. 1998). Taphrina deformans causes host cell elongation and division during colonization of leaf tissue, most likely through the activity of IAA and cytokinins (Sziráki et al. 1975). An IAA synthesis gene is highly expressed in Puccinia graminis f. sp. tritici haustoria during infection of wheat, and silencing of this gene resulted in reduced disease severity (Yin et al. 2014). Beneficial bacteria, such as Azospirillum, Pseudomonas putida, and Rhizobium species, can also synthesize auxin (reviewed in Spaepen et al. 2007). The beneficial fungi, Trichoderma atroviride and Trichoderma virens, produce IAA and related compounds, which along with an intact host auxin signaling/response and transport, modifies host root architecture (Contreras-Cornejo et al. 2009, 2015). There is also evidence to suggest that IAA may act as a signaling molecule for plant-associated bacteria and in the yeast Saccharomyces cerevisiae, as IAA induces changes in microbial gene expression, including virulence genes (reviewed in Kunkel and Harper 2018; Spaepen et al. 2007). 
Plant-associated microbes may also benefit when host auxin signaling/responses and transport are activated. For example, Navarro et al. (2006) reported that over-expression of the IAA receptor auxin signaling F-box 1 (AFB1) protein in the Arabidopsis tirl-1 mutant increased susceptibility to Pseudomonas syringae pv. tomato strain DC3000, while overexpression of the microRNA miR393, which targets transcripts of the transport inhibitor response (TIR1)/AFB receptors, led to increased resistance compared with wild-type plants. Inhibition of auxin transport and modification of auxin signaling/response via miRNA pathways have also been implicated in root nodulation (Rightmyer and Long 2011; Rosendahl and Jochimsen 1995; Roy et al. 2017; Turner et al. 2013). In this case, auxin is involved in initiation of the nodule, vascular patterning, and increasing susceptibility to the colonizing Rhizobia (reviewed in Kohlen et al. 2018; Roy et al. 2017; Shen et al. 2015; Turner et al. 2013). Fusarium oxysporum utilizes several aspects of auxin biology, including biosynthesis, signaling, and transport, to alter auxin homeostasis and to increase susceptibility in Arabidopsis (Kidd et al. 2011). Tryptophan (Trp) biosynthesis genes and auxin signaling and transport genes were also upregulated in two maize near isogenic lines (NILs) during infection with $F$. graminearum and were more highly expressed in the NIL lacking a quantitative disease resistance locus (QDRL) for resistance to this pathogen (Liu et al. 2016). Laccaria bicolor releases an effector that up-regulates auxin signaling/responses to circumvent host defenses (Pieterse et al. 2014; Plett et al. 2011). Auxin transport has also been implicated in susceptibility of Arabidopsis to Phytophthora parasitica and root knot nematode (Evangelisti et al. 2013; Kyndt et al. 2016). When the P. parasitica effector Penetration-specific effector 1 (PSE1) was expressed in Arabidopsis, the Pin-formed (PIN) auxin efflux transport proteins AtPIN4 and AtPIN7 accumulated in additional cell layers at the root apex during growth assays, and the plants were more susceptible (Evangelisti et al. 2013). The pin1, pin2, and pin3 mutants had fewer nematodes and galls compared with wild-type plants infected with root knot nematode, while pin4 mutants had adverse effects on female development and gall size (Kyndt et al. 2016). Additionally, the auxin uptake mutants auxin transporter protein 1 (auxl), like auxl 3 (lax3), and auxllax3 had fewer nematodes and fewer and smaller galls compared with wild-type plants (Kyndt et al. 2016).

The establishment of auxin concentration gradients in plant organs occurs through a combination of metabolism (including biosynthesis and catabolism via conjugation and oxidation) and transport (both intracellular and intercellular). It is a critical component regulating auxin-dependent effects on growth, development, and responses to outside stimuli (Domingo et al. 2009; Korasick et al. 2013; Staswick et al. 2005; Woodward and Bartel 2005; Zhang et al. 2016). IAA is primarily synthesized via two pathways in plants, the Trp-dependent (Soeno et al. 2010; Zhao et al. 2001), and the tryptophan-independent pathway. In Trpdependent IAA biosynthesis, the amino acid Trp is the primary auxin precursor (Fig. 1) (Blakeslee et al. 2019; Korasick et al. 2013; Mashiguchi et al. 2011; Spaepen et al. 2007; Tivendale et al. 2014; Woodward and Bartel 2005). Currently, Trp-independent biosynthesis is poorly defined and is thought to originate from indole or indole-3-glycerol (Korasick et al. 2013; Ouyang et al. 2000; Soeno et al. 2010; Tivendale et al. 2014). Auxin catabolism reduces the amount of active IAA in the cell and is important for maintaining auxin homeostasis (Fig. 1). This occurs primarily either through oxidation or the conjugation of IAA to sugars, amino acids, or proteins. Oxidation of auxin is not reversible and, in most plants, serves as a primary route for auxin catabolism (Korasick et al. 2013; Ludwig-Müller 2011; Woodward and Bartel 2005; Zhang et al. 2016). Conjugation to amino acids is catalyzed by the Gretchen Hagen 3 (GH3) enzymes (Staswick et al. 2005) and can be reversible or irreversible, depending on the specific amino acid moiety added. Examples of reversible amino acid conjugates include indole-3-acetyl leucine (IAA-Leu) and indole-3-acetyl alanine (IAA-Ala). In these compounds, the linkage to the amino acid can be hydrolyzed, releasing free IAA. Therefore, they are often used to store IAA in an inactive form (Korasick et al. 2013; Ludwig-Müller 2011; Woodward and Bartel 2005; Zhang et al. 2016). However, conjugation to other amino acids, such as aspartate and glutamate, which produces indole-3-acetyl-aspartic acid (IAAAsp) and indole-3-acetyl-glutamic acid (IAA-Glu), is not reversible and permanently removes the conjugated IAA moiety to maintain hormone homeostasis within the cell (Korasick et al. 2013; LudwigMüller 2011; Woodward and Bartel 2005; Zhang et al. 2016). IAA or 2-oxindole-3-acetic acid (oxIAA) can also be conjugated to sugars, such as glucose and myo-inositol (Korasick et al. 2013; Ludwig-Müller 2011).

The PIN family is one group of proteins that mediate auxin efflux out of plant cells, and different members are active in different tissues. Based on studies in Arabidopsis, AtPIN1 facilitates transport from the shoot to the roots and plays a role in vascular tissue development, whereas AtPIN2 mediates shootward movement of auxin in root epidermal and cortical cells (Gälweiler et al. 1998; reviewed in Peer et al. 2011). AtPIN2, AUX1, and transient changes in AtPIN1 levels at the cell membrane are involved in root halotropic responses (van den Berg et al. 2016); AUX1 and AtPIN2 also contribute to gravitropism (Luschnig et al. 1998; Müller et al. 1998; reviewed in Peer et al. 2011; Utsuno et al. 1998). AtPIN3 is located in the root pericycle and columella and is involved in gravitropic and phototropic responses (Friml et al. 2002b). AtPIN4 is localized to the quiescent center and nearby cells in the root meristem and is important for proper cell division and development in the root tip (Friml et al. 2002a). Recent studies have profiled soybean homologs of the AtPIN gene family (Liu and Wei 2017; Wang et al. 2015). Wang et al. (2015) found that 15, 8, and 7 of these genes were differentially regulated during drought, salinity, and dehydration stress, respectively. Eighteen and seventeen of these genes responded to abscisic acid (ABA) or IAA treatment, respectively (Wang et al. 2015).

In soybean, modulation of auxin levels has been proposed to play a role in susceptibility of roots to Phytophthora sojae, the causal agent of Phytophthora root and stem rot (Wang et al. 2010, 2012b; Zhou et al. 2009). Three auxin-related genes involved in transport and signaling/response, Glyma.19g128800, an AtPIN1 homolog;

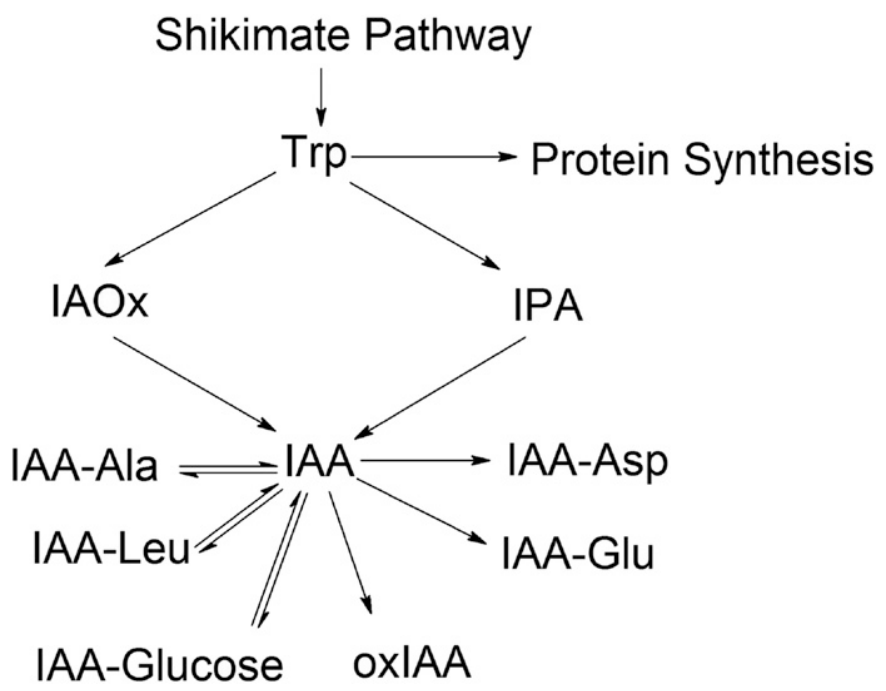

Fig. 1. Overview of tryptophan pathways (Trp) and indole-3-acetic acid (IAA) Trp-dependent biosynthesis and catabolic pathways in plants. indole-3acetaldoxmime (IAOx), indole-3-propionic acid (IPA), indole-3-acetyl-alanine (IAA-Ala), indole-3-acetyl-leucine (IAA-Leu), indole-3-acetyl-aspartic acid (IAA-Asp), indole-3-acetyl-glutamic acid (IAA-Glu), 2-oxindole-3-acetic acid (oxIAA) (Korasick et al. 2013; Mashiguchi et al. 2011; Woodward and Bartel 2005). 
Glyma.19g161000, an auxin-induced gene; and Glyma.19g221900, an auxin-responsive transcription factor, were found to be associated with QDRLs and differentially expressed between a moderately resistant cultivar, Conrad, and a moderately susceptible cultivar, Sloan, following inoculation with $P$. sojae (Wang et al. 2010, 2012b). Further mapping in a larger recombinant inbred line (RIL) population with a higher density of markers identified seven auxin-related genes underlying two QDRLs on chromosome 19 (Stasko et al. 2016). One of these, an auxin/indole-3-acetic acid (Aux/IAA) signaling gene, Glyma.19g181900, was down regulated in root tissue in Sloan following inoculation compared with mock controls beginning at $48 \mathrm{~h}$ postinoculation (hpi) and in the inoculated roots of Conrad compared with mock at 72 hpi in an earlier microarray experiment (Wang et al. 2010; Zhou et al. 2009).

Given the number and types of auxin-related genes underlying the QDRLs for resistance to P. sojae on soybean chromosome 19 (Stasko et al. 2016; Wang et al. 2010, 2012b) and the hormone's role in other plant-microbe interactions, we hypothesized that auxin is involved in $P$. sojae-soybean root interactions. To examine this, we measured auxin (IAA) and auxin-related metabolites in both pathogen and inoculated and mock inoculated plant tissues via liquid chromatography-tandem mass spectrometry (LC-MS/MS). To define possible pathways for auxin synthesis in infected tissues, we investigated for the presence of the IAA precursor Trp and the associated metabolite tryptamine (TRA) in mycelia and synthetic culture media of $P$. sojae. As auxin does not act alone but in coordination with a range of plant hormones, we also profiled a suit of other phytohormones associated with pathogen infection (salicylic acid [SA] and jasmonic acid [JA]) in resistant and susceptible genotypes during a time course assay. A similar time course was used to examine the expression of root-associated soybean GmPIN genes in the same genotypes during infection.

\section{MATERIALS AND METHODS}

$P$. sojae material and tissue collection for auxin metabolite analysis. The mycelia and media supernatant from cultures of $P$. sojae isolates $\mathrm{OH} 1, \mathrm{OH} 4, \mathrm{OH} 7, \mathrm{OH} 25$, and $\mathrm{OH} 12168-05-01$ were analyzed for IAA and auxin metabolites in two separate experiments, the first conducted in the fall of 2016 and the second during the winter of 2017. In the first experiment, five colonized lima bean agar plugs of approximately $5 \times 5 \mathrm{~mm}$ in size from each isolate were placed in separate flasks containing $50 \mathrm{ml}$ of a synthetic medium (2.4 g of sucrose, $273 \mathrm{mg}$ of asparagine, $152 \mathrm{mg}$ of $\mathrm{KH}_{2} \mathrm{PO}_{4}$, $151.2 \mathrm{mg}$ of $\mathrm{K}_{2} \mathrm{HPO}_{4}, 11 \mathrm{mg}$ of cholesterol, $11.2 \mathrm{mg}$ of ascorbic acid, $5.9 \mu \mathrm{M}$ thiamine $\mathrm{HCl}, 15.3 \mu \mathrm{MZnSO}_{4} \cdot 7 \mathrm{H}_{2} \mathrm{O}, 3.6 \mu \mathrm{M} \mathrm{FeSO}_{4}$. $7 \mathrm{H}_{2} \mathrm{O}$, and $0.3 \mu \mathrm{M} \mathrm{MnCl}{ }_{2} \cdot 4 \mathrm{H}_{2} \mathrm{O}$ ) (Hoitink and Schmitthenner 1969; Qutob et al. 2000). Four flasks were prepared for each isolate and placed in a cabinet at room temperature (approximately 24 to $26^{\circ} \mathrm{C}$ ) for 1 week. Each flask was considered one biological replicate. Mycelia and media were carefully transferred to a Büchner funnel with an 11.0-cm-diameter Whatman No. 5 nitrocellulose disc filter placed in the bottom, and the liquid medium was separated from the mycelia using vacuum filtration. The four replicates for each isolate were pooled into a single sample to ensure that the quantity of mycelia was suitable for auxin metabolite extraction. The agar plugs were removed, and the mycelia were transferred to a 2-ml microcentrifuge tube. The tube was wrapped in aluminum foil, immediately frozen in liquid nitrogen, and stored at $-80^{\circ} \mathrm{C}$ until metabolite analyses. Ten milliliters of the filtered minimal medium used to grow each isolate was also collected in separate $50-\mathrm{ml}$ conical centrifuge tubes. These tubes were wrapped in foil and immediately stored at $-80^{\circ} \mathrm{C}$. In the second experiment, the same isolates were grown in $25 \mathrm{ml}$ of synthetic medium to increase the surface area for the cultures and to concentrate any secretions from the mycelia into the media. Once again, four biological replicates, consisting of one flask per replicate, were prepared for each isolate. Samples were once again pooled by isolate and collected as described above, including $10 \mathrm{ml}$ of filtered medium without any $P$. sojae growth, which served as a negative control.

Plant material and tissue collection for auxin metabolite analysis. Two experiments to evaluate the accumulation of auxin and other metabolites in root tissue during inoculation with $P$. sojae were conducted in summer of 2015 followed by a second in winter of 2018. Seeds of the moderately resistant cultivar Conrad and the moderately susceptible cultivar Sloan were sterilized with chlorine gas as previously described (Govindarajulu et al. 2008, 2009) to remove any seedborne organisms. Briefly, Petri plates with a single layer of seed were placed in a vacuum desiccator in a fume hood with a $250-\mathrm{ml}$ beaker containing $100 \mathrm{ml}$ of sodium hypochlorate. One milliliter of reagent grade hydrochloric acid $(\mathrm{HCl})$ was added to the beaker. A vacuum was applied to the desiccator for about $5 \mathrm{~min}$, after which the vacuum was stopped, and the beaker was stored overnight. Soybean plants of both cultivars were inoculated by means of a modified tray test (Wang et al. 2010, 2012a, b) in two separate experiments. Fifteen to twenty sterilized seeds of Conrad and Sloan were planted in fine vermiculite (Perlite Vermiculite Packing Industries, Inc., North Bloomfield, $\mathrm{OH}$ ) in 0.5-liter polystyrene cups and placed in a greenhouse in the first experiment. To better control for environmental variability, plants in the second experiment were placed in a growth chamber at $25^{\circ} \mathrm{C}$ with $16 \mathrm{~h}$ light. After 7 days, plants were removed from the cups, and the roots were gently washed in tap water. Eight to ten plants were then placed on a polyester cloth on top of a cotton wicking pad on a plastic tray. Each tray represented one replication, and there were three replications per cultivar, treatment, and time point in each experiment.

Preparation of zoospore inoculum of $P$. sojae was modified from Qutob et al. (2000) and Mideros et al. (2007). Briefly, 3-day-old cultures of $P$. sojae $\mathrm{OH} 25$ grown on $\mathrm{V} 8$ juice agar were flooded with $15 \mathrm{ml}$ of sterile, distilled $\mathrm{H}_{2} \mathrm{O}$ ( $\mathrm{pH} 6.8$ to 7.1 ) for 14 to $16 \mathrm{~h}$. The water was then replaced with fresh water every $30 \mathrm{~min}$ for a total of six times and then was replaced again after $3 \mathrm{~h}$. Finally, plates were incubated for an additional $15 \mathrm{~h}$ at $25^{\circ} \mathrm{C}$ to increase the concentration of zoospores. A sterile paper towel strip (approximately 0.5 to $1.0 \mathrm{~cm}$ wide and $25.5 \mathrm{~cm}$ long) was placed under the roots, $4 \mathrm{~cm}$ from the crown region. A 100- $\mu$ l aliquot of the zoospore suspension, $0.5 \times 10^{4}$ to $0.9 \times 10^{4}$ zoospores $/ \mathrm{ml}$, was then pipetted onto the strip to the immediate right of the main tap root of each plant. A second sterile paper towel strip was placed over the roots at the same location, and an additional $100 \mu \mathrm{l}$ of zoospores was pipetted onto the strip directly over the main tap root for a total of approximately 1,000 to 1,800 zoospores per plant. This was done to ensure that the root was inoculated from both sides and to reduce tissue dilution effects. For the mock-inoculated plants, $100 \mu \mathrm{l}$ of sterile, distilled water (pH 6.8 to 7.1) was used on each strip in place of the zoospore suspension. Trays were stacked by time point to minimize the manipulation of the plants and were kept in a growth chamber as described above.

Root sections $(1 \mathrm{~cm})$ were collected from each plant at the inoculation site at $0,12,24$, and $48 \mathrm{hpi}$ and from the upper edge of the lesion margin at $72 \mathrm{hpi}$ in the first experiment. In the second experiment, trays were drained for approximately $1 \mathrm{~h}$ prior to tissue collection to remove excess water. Tissue was collected as described for the first experiment, but the 12 hpi time point was dropped. In both experiments, lateral roots were removed from the collection site. Sections from 7 to 10 plants from each tray were pooled into one sample, excluding plants that had potential secondary infections. Three replicates were collected at each time point for each treatment for a total of 24 to 30 plants per cultivar. Root tissue samples were frozen and stored as described above for the mycelial samples.

To ensure that the inoculation was successful and that there was a significant difference between the two cultivars, additional trays of each cultivar were either inoculated or mock inoculated and kept 
until 7 days postinoculation (dpi) in the growth chamber at the same conditions as described above. The lesion length was measured on each plant from the top of the inoculation site to the upper edge of the lesion margin. Individual plants with no visible lesions were treated as missing data to account for infection efficiency. The lesion lengths were then analyzed by analysis of variance using PROC GLM followed by Fisher's protected least significant difference (LSD) in SAS v9.4 (SAS Institute Inc., Cary, NC). The lesion lengths of individual plants were included in the SAS model due to the level of specificity needed in these assays.

Auxin metabolite extraction and quantification. Auxin metabolites were extracted and purified using a protocol modified from Blakeslee and Murphy (2016). Mycelia in both P. sojae experiments or roots in the first inoculated soybean experiment were finely ground with plastic pestles in 1.5 - to $2.0-\mathrm{ml}$ microcentrifuge tubes. The tubes were kept in liquid nitrogen during the grinding process to prevent the tissue from thawing. Ground tissue was suspended in $1 \mathrm{ml}$ of $50 \mathrm{mM}$ sodium phosphate buffer $(\mathrm{pH} 7.0$, $1 \%$ diethyldithiocarbamate, DETC). Internal standards (Supplementary Table S1) were then added to each tube. Roots were weighed in a $2-\mathrm{ml}$ microcentrifuge tube.

Due to the fibrous nature of the roots and to improve sample throughput, the plant material in the second experiment was homogenized using a Mixer Mill 301(Retsch, Hann, Germany) and metal beads. In these assays, the root tissue was first broken into approximately 2- to 4-mm pieces in liquid nitrogen using a mortar and pestle. Root fragments were then transferred to a 2-ml microcentrifuge tube containing two 4-mm-diameter metal beads. Microcentrifuge tubes were placed in a prechilled aluminum block in a liquid nitrogen bath. To homogenize tissues, aluminum blocks were loaded into the Mixer Mill and shaken at $20 \mathrm{~Hz}, 4^{\circ} \mathrm{C}$ for $2 \mathrm{~min}$. This process was repeated, after which $1 \mathrm{ml}$ of sample buffer and internal standards were added to each sample.

In all experiments, auxin metabolites were extracted from tissues by shaking the tubes containing ground tissue on a Biomixer lab nutator (Benchmark Scientific, Edison, NJ) at $4^{\circ} \mathrm{C}$ for $20 \mathrm{~min}$. Samples were then centrifuged at $12,000 \times g$ at $4^{\circ} \mathrm{C}$ for $15 \mathrm{~min}$. The supernatant was transferred to a new tube, and the $\mathrm{pH}$ was adjusted to 3.0 with $1 \mathrm{~N} \mathrm{HCl}$. Auxin metabolites were purified on an Oasis HLB solid phase extraction column (Waters Corp, Milford, MA) at $4^{\circ} \mathrm{C}$. The column was conditioned with $1 \mathrm{ml}$ methanol, followed by $1 \mathrm{ml}$ of water, and then by $0.5 \mathrm{ml}$ of $50 \mathrm{mM}$ sodium phosphate buffer ( $\mathrm{pH}$ 2.7). After conditioning, the sample was run through the column, which was then washed with $5 \%$ methanol and eluted with $80 \%$ methanol. In the second inoculated root experiment, column steps were sped up by using a low-pressure system to feed buffer and samples through the columns. In all experiments, samples were dried under nitrogen gas and redissolved in $100 \%$ methanol. For LC-MS/MS analyses, samples were injected (injection volume of $0.4 \mu \mathrm{l}$ for IAA analyses or $1 \mu \mathrm{l}$ for SA and JA analyses) into an Agilent 6460 triple quadrupole LC-MS/MS system (Agilent Technologies), as described previously (Blakeslee and Murphy 2016; Zhang et al. 2018).

For the synthetic media samples, $5 \mathrm{ml}$ of the noncolonized or colonized media supernatant were transferred to a new 15-ml tube. One milliliter of sodium phosphate buffer $(\mathrm{pH} 2.7)$ and the internal standards from above were added to each tube. The $\mathrm{pH}$ of the samples was adjusted to 3.0 with $1 \mathrm{~N} \mathrm{HCl}$. Metabolites were then purified using solid-phase extraction and analyzed as described above.

Raw LC-MS/MS data were processed using MassHunter software (Agilent Technologies). Samples that had a signal to noise ratio less than three were considered below the limit of detection (LOD), the minimum concentration at which the instrument can reliably detect the compound. Samples with a signal to noise ratio between 3.0 and 10.0 were considered above LOD but below limit of quantification (LOQ), the minimum concentration at which the compound can be reliably quantified. Samples with a signal to noise ratio above 10.0 were considered above LOQ. The total concentration of each compound was adjusted for the sample weight and injection volume. For statistical analysis of the soybean root experiments, samples that were below the LOD were set to a concentration of $0 \mathrm{ng} / \mathrm{g}$ frozen weight in the first experiment. In the second experiment, values were set to $0.001 \mathrm{ng} / \mathrm{g}$ frozen weight. Significant differences in the inoculated compared with the mock roots within cultivar at a given time were determined using Student's $t$ test. Significance across all treatments within a time point were determined using PROC GLM followed by Tukey's honest significant difference (HSD) in SAS.

Quantitative real-time PCR (qRT-PCR) of GmPIN genes. Tap root samples of Conrad and Sloan inoculated with zoospores of $P$. sojae isolate $\mathrm{OH} 25$ or mock inoculated with sterile distilled water were obtained following the protocol described above for extraction and quantification of auxin metabolites. For the gene expression analysis, the experimental design consisted of a randomized complete block design with a factorial: cultivar (Conrad and Sloan) by time point $(24,48$, and $72 \mathrm{hpi}$ ) by treatment (mock and inoculated). The experiment was replicated once, and each replicated experiment had four biological reps. Tissue from two biological reps within each experiment of a given cultivar and treatment within each time point were pooled for RNA extraction to increase the RNA concentration. RNA was extracted using the QIAGEN RNeasy Plant Mini Prep Kit (Qiagen, Valencia, CA) with the optional $\beta$-mercapto-ethanol treatment. RNA quality was assessed on a ND-1000 Nanodrop (Nanodrop Technologies, Wilmington, DE) and by electrophoresis on a $1 \%$ agarose gel. The RNA concentration was determined using the QubitTM RNA BR Assay kit (Invitrogen Inc., Carlsbad, CA) on a Qubit 2.0 Flourometer. For cDNA synthesis, $1 \mu \mathrm{g}$ of RNA was reverse transcribed with the SuperScript III kit (Invitrogen Inc.). Eleven GmPIN genes and one reference gene (Table 1) were used for qRTPCR. The GmPIN genes were selected based on their homology to Arabidopsis PIN proteins or their expression in soybean roots (Wang et al. 2010; Wang et al. 2015). The reference ubiquitin gene, GmUBI3 (Chai et al. 2015; Wang et al. 2015), showed consistent and stable expression across cDNA samples compared with two other reference genes, cons6 (a F-box gene) and cons7 (a metalloprotease; Libault et al. 2008). Primers (Table 2) were checked for product specificity by BLASTn search against the soybean genome (Wm82.a2.v1) at both Phytozome v.12.1 (https://phytozome.jgi. doe.gov) and SoyBase (Grant et al. 2010). qRT-PCR was performed with three technical reps in a CFX-384 Real-time System on a C1000 touch thermocycler (Bio-Rad, Hercules, CA) using the SYBR/fam detection mode. Reaction conditions were as follows: $95^{\circ} \mathrm{C}$ for $3 \mathrm{~min}$, 35 cycles of $95^{\circ} \mathrm{C}$ for $10 \mathrm{~s}$ and $60^{\circ} \mathrm{C}$ for $45 \mathrm{~s}$ followed by $95^{\circ} \mathrm{C}$ for $1 \mathrm{~min}$ and $60^{\circ} \mathrm{C}$ for $1 \mathrm{~min} 31 \mathrm{~s}$. Normalized gene expression was obtained using the Bio-Rad CFX Maestro software (http://www.bio$\mathrm{rad} . \mathrm{com} / \mathrm{webroot} / \mathrm{web} / \mathrm{pdf} / \mathrm{lsr} /$ literature/10000068703.pdf). Significant differences in gene expression of the inoculated compared with the mock within cultivar at a given time point were determined using Student's $t$ test. Significance across all treatments within a time point were determined using PROC GLM followed by Tukey's HSD in SAS.

\section{RESULTS}

Auxinic metabolites in $P$. sojae mycelia and associated media. To assess whether different isolates of $P$. sojae could be a source of IAA, mycelia and media supernatant were evaluated for the presence of Trp and TRA via LC-MS/MS in two separate experiments. As expected for an amino acid essential for cellular protein synthesis, Trp was detected in the mycelia of all isolates in both experiments, and its concentrations ranged from 3,405.15 to 16,347.81 ng/g (Fig. 2). 
Trp and TRA were also detected in the media supernatant from all of the isolates in both experiments, at concentrations ranging from 591.93 to $1,424.65 \mathrm{ng} / \mathrm{liter}$ and from 7.22 to $56.22 \mathrm{ng} / \mathrm{liter}$, respectively (Figs. 3 and 4). While trace amounts of TRA were detected in the negative control in the second experiment, levels of this compound were well below the LOQ but above the LOD (Fig. 4B). IAA was not consistently above LOQ in both experiments. The presence of Trp in the media supernatant suggests that $P$. sojae may secrete this compound into its surroundings. However, as careful as the extraction process was, the potential for Trp release into the media as a result of the lysis of mycelia cannot be ruled out.

Quantification of auxin metabolites in soybean root tissue during infection with $P$. sojae. To verify that the zoospore inoculations were successful for the time course series, lesions were measured at $7 \mathrm{dpi}$. For all of these experiments, the adjusted mean lesion length was significantly different between Conrad and Sloan $(P<0.001 ;$ Fig. 5), indicating that the inoculations were successful.

In both experiments, changes in the levels of Trp, IAA, indole-3acetyl-glutamic acid (IAA-Glu), IAA-Ala, SA, and JA (Fig. 6) were observed in both cultivars following inoculation. While similar trends in phytohormone levels were, in general, observed between the two studies, the absolute values of each individual metabolite varied considerably between experiments. This variation is expected and most likely due to differences in plant growth and development and in infection stage between the two studies, as evidenced by the differences in lesion lengths between the two experiments (Fig. 5). While the shift in extraction methodology used in the second study could also contribute, this is less likely as analyses from the LC-MS/MS indicated similar percent recovery of internal standards.

In the first experiment, none of the metabolite levels were significantly different between the two cultivars or between the mock and inoculated treatment within cultivar at $12 \mathrm{hpi}$; therefore, this time point was dropped in the second experiment. Based on the initial experiments indicating that $P$. sojae may release Trp into infected roots, levels of this auxin precursor were measured in root tissues following inoculation. In the first experiment, Trp was higher in Sloan inoculated roots than mock roots by Student's $t$ test and higher than levels in Conrad inoculated roots by Tukey's HSD at 48 hpi (Fig. 6A). In the second experiment, levels of Trp were significantly higher in the inoculated samples compared with their mock controls in both cultivars at 72 hpi (Fig. 6B). As expected, based on the increased levels of Trp observed after inoculation, IAA levels were also significantly higher in Sloan inoculated roots than all others at $48 \mathrm{hpi}$ in the first experiment (Fig. 6C). Similarly, in the second experiment, IAA levels were significantly higher in Sloan inoculated compared with mock roots, although in this assay the difference occurred at 72 hpi (Fig. 6D). Likewise, IAA-Glu was significantly higher in Conrad inoculated roots compared with the mock at 48 hpi and was significantly higher in inoculated roots compared with mock roots of both cultivars by Student's $t$ test at $72 \mathrm{hpi}$ in the first experiment (Fig. 6E). In the second experiment, levels of IAA-Glu were significantly higher in Sloan inoculated compared with the mock inoculated roots of both cultivars at $48 \mathrm{hpi}$ and higher than all other roots at $72 \mathrm{hpi}$ (Fig. 6F). IAA-Ala levels were significantly higher in Sloan inoculated roots than in either Conrad treatment at $72 \mathrm{hpi}$ in both experiments and were higher compared with Sloan mock roots in the second experiment (Fig. $6 \mathrm{G}$ to $\mathrm{H})$. Thus, there was an overall increase in IAA, its precursor, and the amino acid conjugates IAA-Glu and IAA-Ala in Sloan inoculated roots during the later stages of infection, which corresponds to the beginning of necrosis in this moderately susceptible cultivar.

As noted above, IAA does not function in a vacuum in plant-pathogen interactions but instead acts in and against a background of additional plant hormones. To better define the role of auxin in the totality of pathogen-induced hormone signaling, we also quantified SA and JA, which have both been associated with

TABLE 1. GmPIN genes investigated in this study and their nearest Arabidopsis homolog

\begin{tabular}{|c|c|c|c|c|}
\hline Gene $^{\mathrm{a}}$ & Locus ID (Wm82.a2/Wm82.a1.v1.1) & Chromosome & Predicted protein length (aa) ${ }^{\mathrm{a}}$ & Arabidopsis homolog ${ }^{\mathrm{a}}$ \\
\hline GmPIN1a & Glyma.08g054700/Glyma08g05900 & $\mathrm{Gm} 08$ & 603 & AtPIN1 \\
\hline GmPIN1b & Glyma.07g102500/Glyma07g11550 & Gm07 & 605 & AtPIN1 \\
\hline GmPINlc ${ }^{\mathrm{b}}$ & Glyma09g30700 & Gm09 & 605 & AtPIN1 \\
\hline GmPINle & Glyma.19g128800/Glyma19g30900 & Gm19 & 578 & AtPIN1 \\
\hline GmPIN2a & Glyma.13g101900/Glyma13g00390 & Gm13 & 642 & AtPIN2 \\
\hline GmPIN2b & Glyma.17g057300/Glyma17g06460 & Gm17 & 637 & AtPIN2 \\
\hline GmPIN3a & Glyma.07g217900/Glyma07g34190 & Gm07 & 665 & AtPIN3/AtPIN4/AtPIN7 \\
\hline GmPIN9d & Glyma.15g208600/Glyma15g25690 & Gm15 & 492 & None \\
\hline
\end{tabular}

a According to Wang et al. (2015).

${ }^{\mathrm{b}}$ No corresponding Locus ID available in Wm82.a2 version of the soybean genome.

TABLE 2. Quantitative real-time PCR primers used in this study and their efficiencies

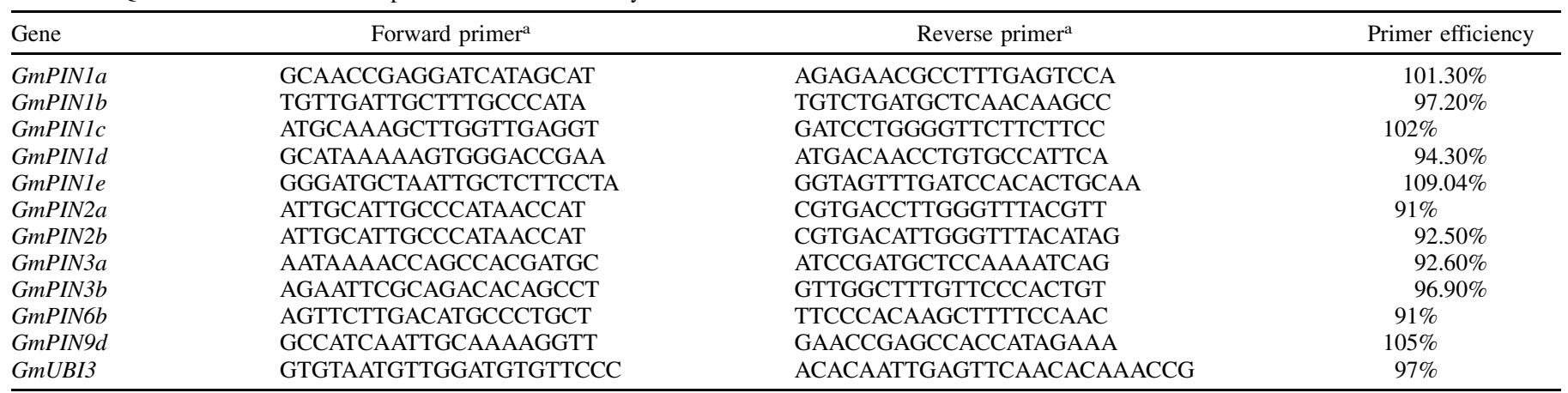

a GmPIN primers from Wang et al. (2015). GmUBI3 primers from Chai et al. (2015) and Wang et al. (2015). 
pathogen infection (Glazebrook 2005; Park et al. 2007; Pieterse et al. 2012; Robert-Seilaniantz et al. 2011; Vlot et al. 2009). In the first experiment, SA levels were significantly higher in Conrad inoculated roots compared with the mock treatment at $24 \mathrm{hpi}$ (Fig. 6I). However, in the second experiment, it was higher in the Sloan inoculated roots at this same time point (Fig. 6J). At 48 hpi, SA levels were significantly higher in inoculated roots of both cultivars compared with their respective controls in the first experiment (Fig. 6I). This trend continued for Sloan in the second experiment (Fig. $6 \mathrm{~J})$. In the first experiment, JA levels were significantly higher in the inoculated roots of both cultivars at $48 \mathrm{hpi}$ (Fig. 6K). This continued for the Sloan inoculated roots in the second experiment (Fig. 6L). JA levels were also significantly higher in Conrad inoculated roots compared with its mock control at 72 hpi in both experiments and were significantly higher in the Sloan inoculated roots than all mock treatments in the second experiment (Fig. $6 \mathrm{~K}$ to $\mathrm{L}$ ).

Expression of GmPIN genes. Similar to the results observed in metabolite quantification studies, gene expression tray tests showed a highly significant difference $(P<0.001)$ in lesion length between Conrad and Sloan inoculated plants in both experiments (Supplementary Fig. S1). Therefore, inoculation with zoospores was successful.

All 11 GmPINs were expressed in the roots of both cultivars at all time points examined (Figs. 7 and 8). GmPINla had significantly higher expression in Conrad inoculated roots compared with the mock treatment and compared with Sloan mock or inoculated roots at 24 hpi in the second experiment (Fig. 7B). While not statistically significant, expression of GmPINla was higher in Conrad inoculated

\section{Tryptophan in Phytophthora sojae Mycelia}
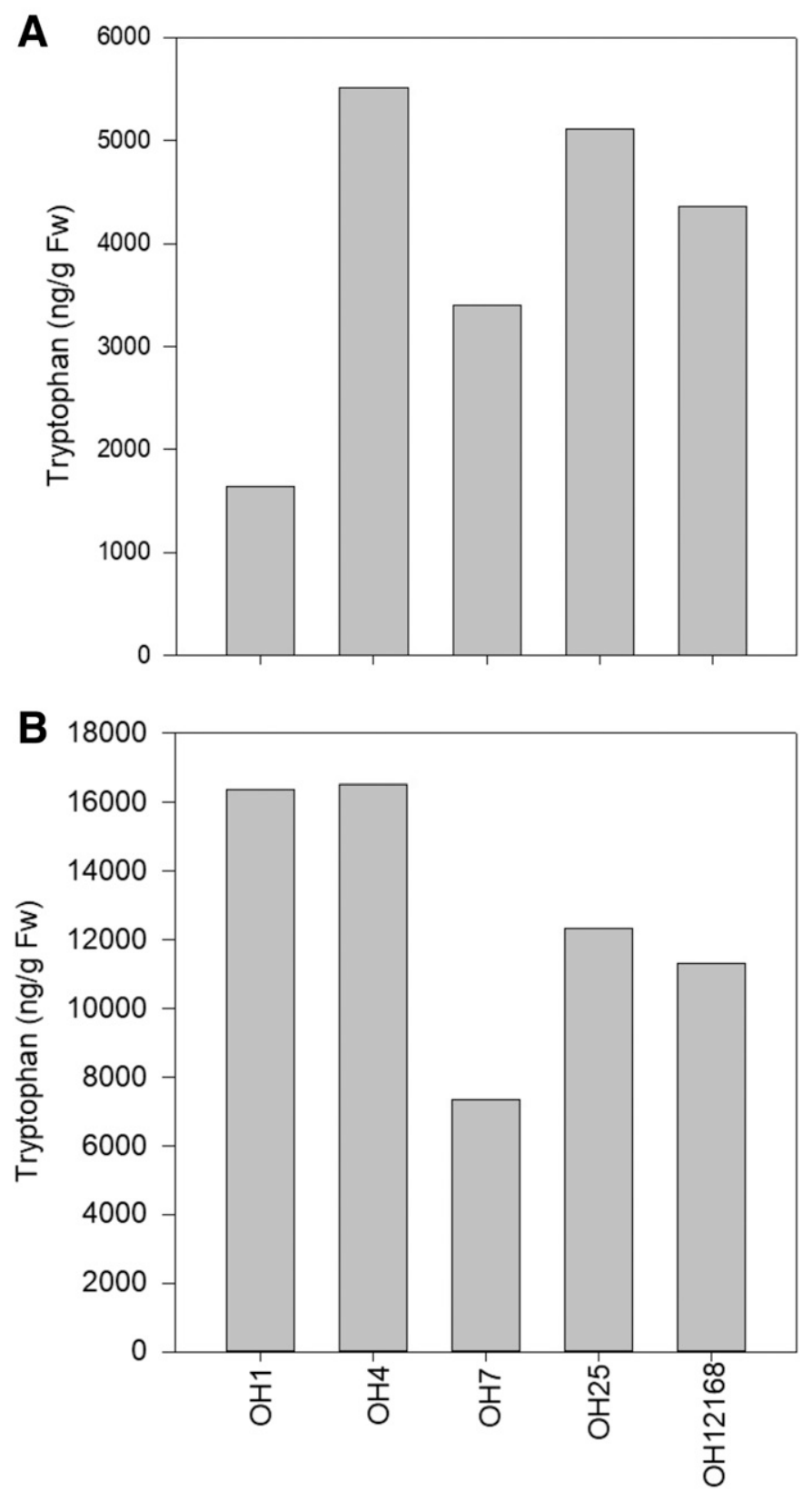

Phytophthora sojae Isolate

Fig. 2. Levels (ng/g frozen weight [Fw]) of tryptophan (Trp) in mycelia of five isolates of Phytophthora sojae in A, experiment one and $\mathbf{B}$, experiment two. Trp levels in the media-only control for experiment two were below limit of detection.
Tryptophan in Phytophthora sojae Media Supernatant
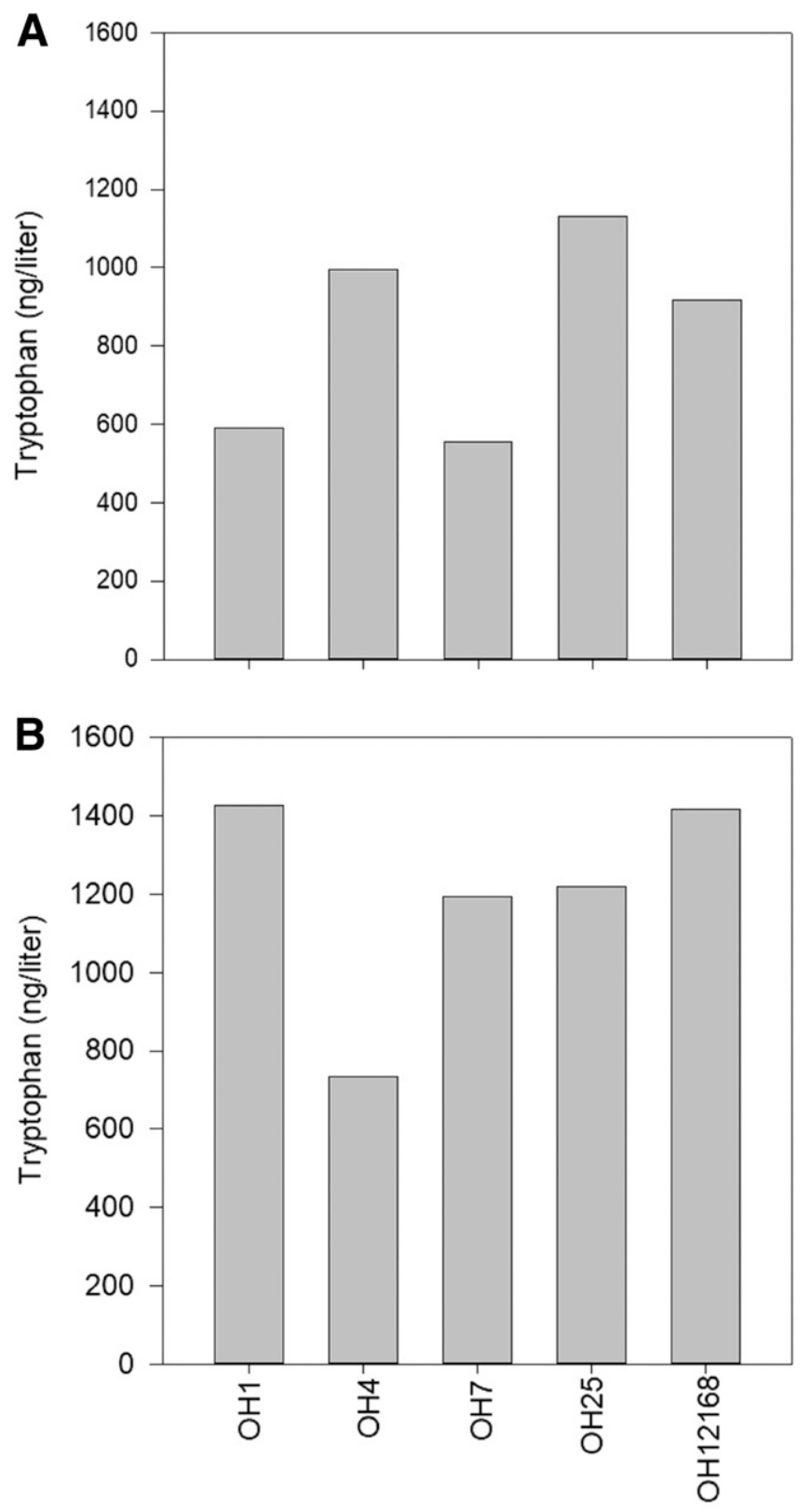

Phytophthora sojae Isolate

Fig. 3. Levels (ng/liter) of tryptophan (Trp) in media supernatant of five Phytophthora sojae isolates in $\mathbf{A}$, experiment one and $\mathbf{B}$, experiment two. Trp levels in the media-only control in experiment two were below the limit of detection. 
roots compared with mock controls in the first experiment (Fig. 7A). This gene had its highest expression in Conrad inoculated roots at $24 \mathrm{hpi}$ and increased in expression in Sloan inoculated roots between 48 and 72 hpi in both experiments (Fig. 7A and B). The expression of GmPIN1b was significantly higher in Conrad inoculated roots at $72 \mathrm{hpi}$ in both experiments compared with all

\section{Tryptamine in Phytophthora sojae Media Supernatant}
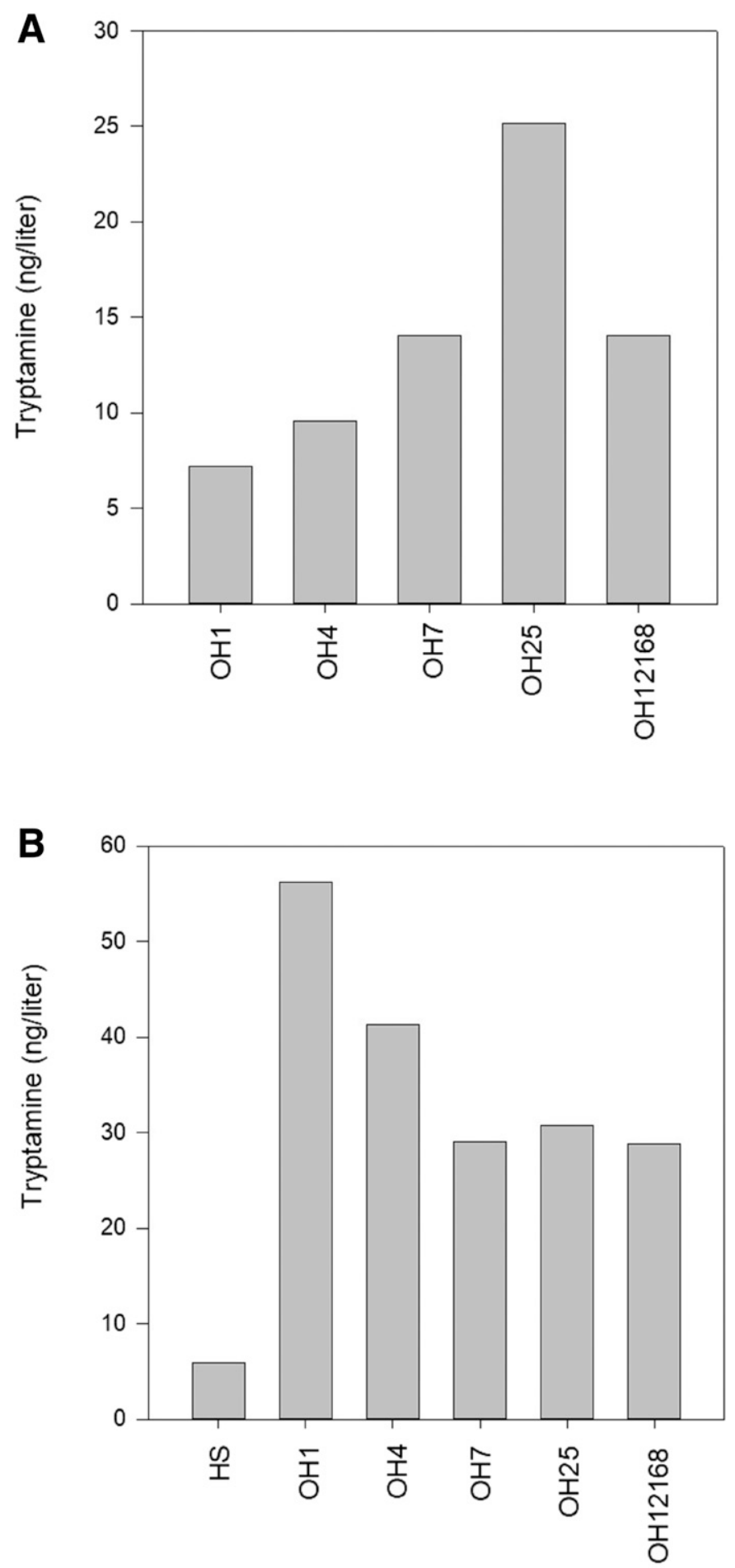

\section{Phytophthora sojae isolate}

Fig. 4. Levels (ng/liter) of tryptamine in media supernatant of five Phytophthora sojae isolates in A, experiment one and $\mathbf{B}$, experiment two. HS, the media-only control, was only included in the second experiment. other treatments. In that same treatment, expression decreased between 24 and 48 hpi before reaching its highest level of expression at 72 hpi. GmPIN1b expression was higher in Sloan inoculated roots compared with mock controls at 24 and 72 hpi in both experiments, but this was not statistically significant (Fig. 7C and D). GmPINle was significantly higher in the inoculated compared with mock treatments in both cultivars at $72 \mathrm{hpi}$, with Conrad inoculated roots having significantly higher expression than Sloan inoculated roots in the first experiment (Fig. 7I). While not statistically significant, GmPINle had higher expression in Conrad inoculated compared with Sloan inoculated roots in the second experiment (Fig. 7J).
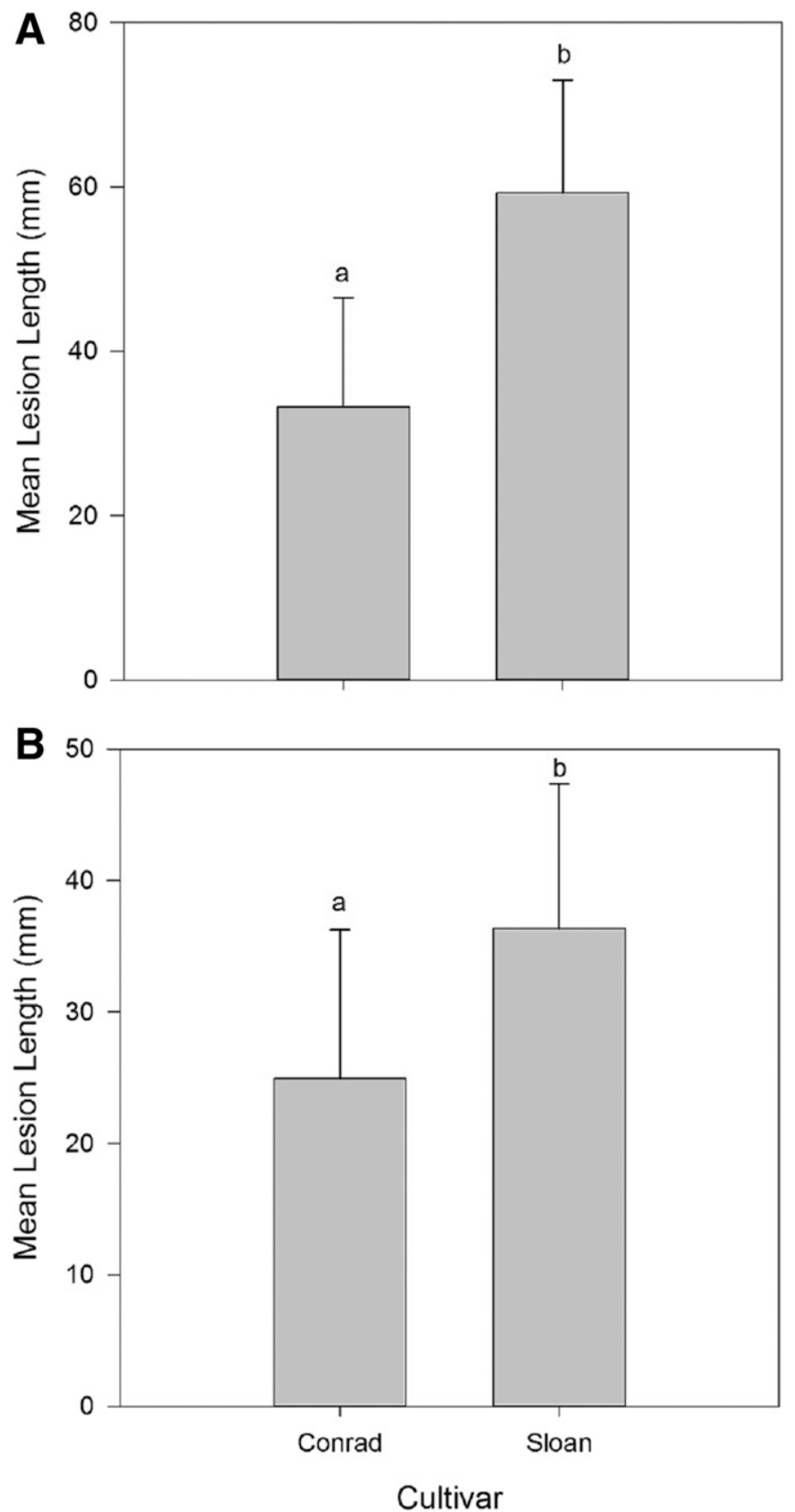

Fig. 5. Mean lesion length of Conrad and Sloan in metabolite tray tests 7 days postinoculation with Phytophthora sojae in $\mathbf{A}$, experiment one and $\mathbf{B}$, experiment two. Means are of two to three biological replicates with 8 to 10 seedlings per replicate and one lesion per seedling. Error bars represent standard deviation. Bars with different letters are significantly different from each other according to Fisher's protected least significant difference $(P<$ $0.001)$. 
GmPIN2a had significantly higher expression in Conrad inoculated roots compared with mock controls at $72 \mathrm{hpi}$ in the second experiment (Fig. 8B). Though not significant, this same trend was observed in the first experiment (Fig. 8A). Additionally, expression levels of this gene increased in Conrad inoculated roots from 48 to
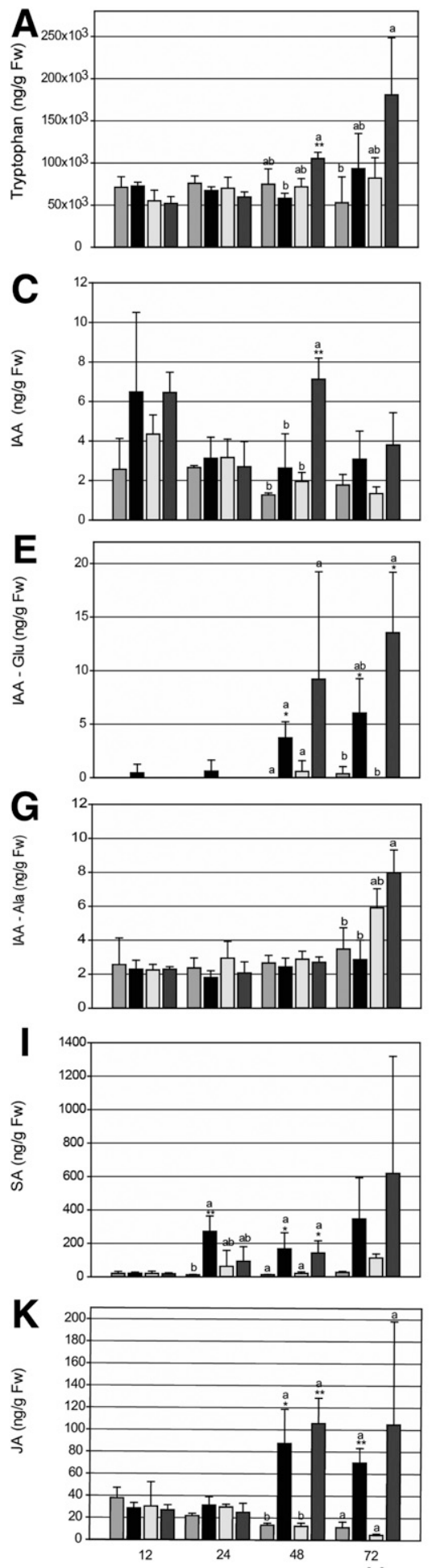

Hours post inoculation (hpi)

$\square$ Conrad Mock $\square$ Conrad Inoculated $\square$ Sloan Mock

H

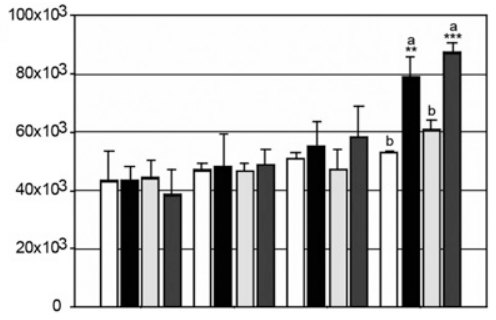

D

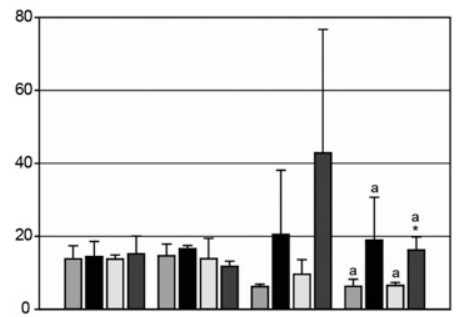

$\mathbf{F}$
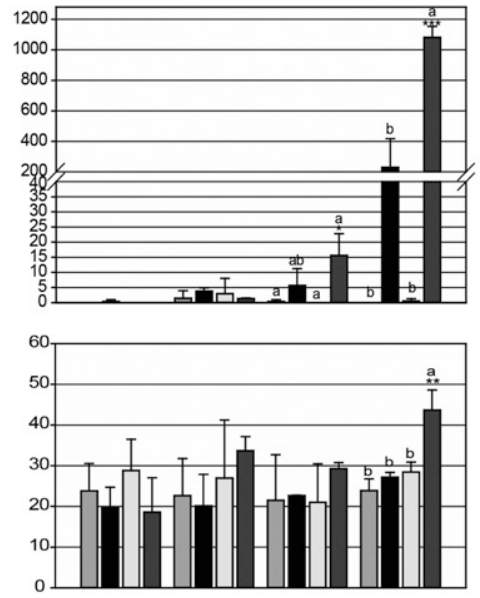

J

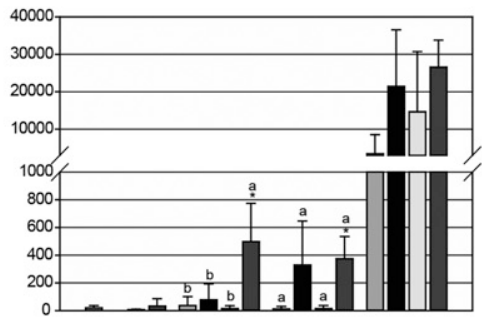

L

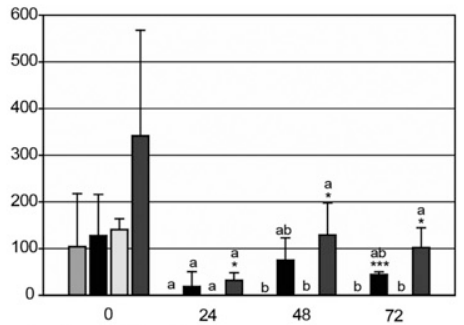

Fig. 6. Mean levels (ng/g frozen weight [Fw]) of $\mathbf{A}$ and B, tryptophan, $\mathbf{C}$ and D, indole-3-acetic acid (IAA), $\mathbf{E}$ and F, indole-3-acetyl-glutamic acid (IAA-Glu), $\mathbf{G}$ and $\mathbf{H}$, indole-3-acetyl-alanine (IAA-Ala), $\mathbf{I}$ and $\mathbf{J}$, salicylic acid (SA), and $\mathbf{K}$ and $\mathbf{L}$, jasmonic acid (JA) in Conrad and Sloan mock and inoculated root tissue. Levels are shown at 12, 24, 48, and 72 at h postinoculation (hpi) in experiment one (A, C, E, G, I, and K) and 0, 24, 48, and 72 hpi in experiment two (B, D, F, H, J, and L). Bars with different letters are significantly different from each other within a time point by Tukey's honest significant difference at $95 \%$ confidence. Asterisks indicate significant difference between mock and inoculated treatments within cultivar at a given time point by Student's $t$ test $(*, P<0.05$; **, $P<0.01$; and $* * *, P<0.001)$. Means are of two to three biological replicates. Error bars represent standard deviation. 
Expression levels of GmPIN1c, GmPIN1d, GmPIN2b, and GmPIN3a varied between the two experiments(Figs. 7E to $\mathrm{H}$ and $8 \mathrm{C}$ to $\mathrm{F}$ ). While not significant, the expression of GmPIN1c increased in Sloan inoculated roots between 48 and 72 hpi in both experiments (Fig. 7E and F). Expression of GmPIN3a was significantly higher in Conrad inoculated roots than in all other
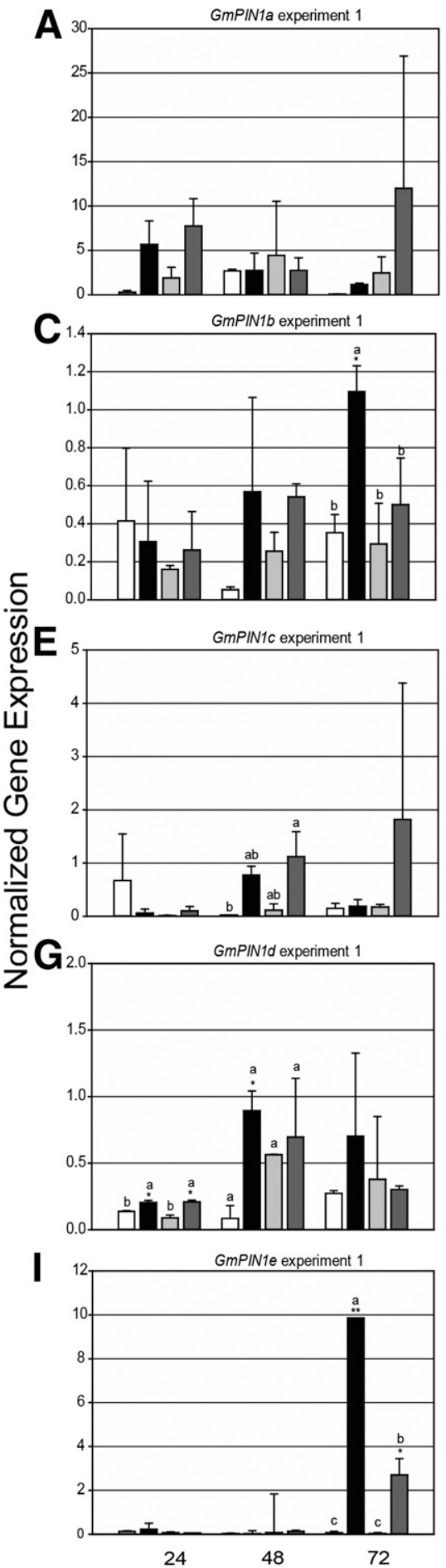

treatments at 24 hpi in the second experiment (Fig. 8F). However, there were no significant differences in expression of this gene in the first experiment or at the other time points in the second experiment (Fig. 8E and F). Expression of GmPIN2 $b$ was significantly higher in Conrad mock than inoculated roots at $72 \mathrm{hpi}$ in the first experiment (Fig. 8C). However, there were no significant differences in the

B

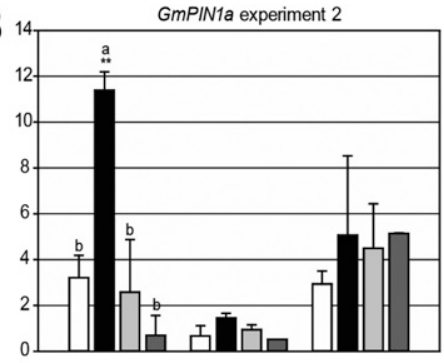

D

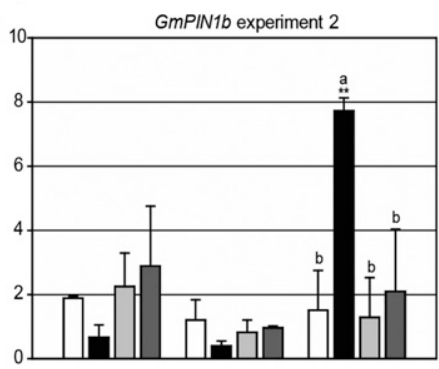

$\mathbf{F}_{2}$

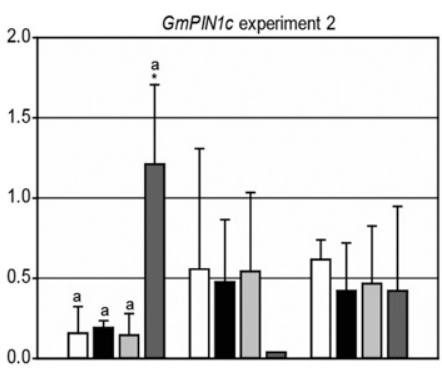

H

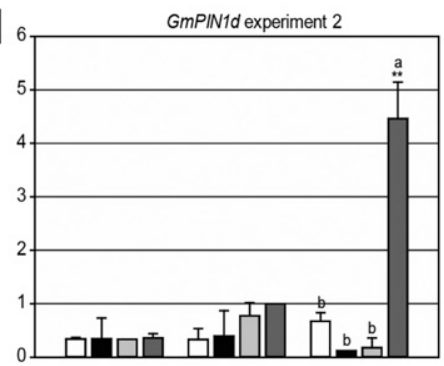

J

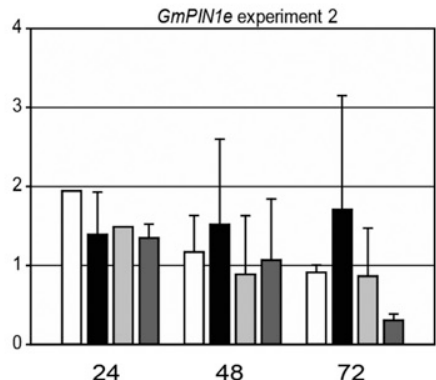

Hours post inoculation (hpi)

Conrad Mock

Conrad Inoculated $\square$ Sloan Mock $\square$ Sloan Inoculated

Fig. 7. Changes in gene expression of A and B, GmPINla (Glyma.08g054700), C and D, GmPIN1b (Glyma.07g102500), E and F, GmPIN1c (Glyma09g30700), $\mathbf{G}$ and H, GmPIN1d (Glyma.03g126000), and I and J, GmPIN1e (Glyma.19g128800) relative to GmUBI3 (Chai et al. 2015; Wang et al. 2015) in Conrad and Sloan mock or inoculated roots in two independent experiments. Bars with different letters are significantly different from each other within a time point by Tukey's honest significant difference at $95 \%$ confidence. Asterisks indicate significant difference between mock and inoculated within cultivar at a given time point by Student's $t$ test $(*, P<0.05$; **, $P<0.01$; and ***, $P<0.001)$. Data represent means of two biological reps with three technical reps. Error bars represent standard deviation. 
second experiment (Fig. 8D). GmPINld expression had no clear trends between the two experiments (Fig. $7 \mathrm{G}$ and H). Finally, $G m P I N 3 b$ and GmPIN9d had no significant differences in expression for any cultivar/treatment combination at all three time points (Fig. $8 \mathrm{G}$ and $\mathrm{H}$, and $\mathrm{K}$ and $\mathrm{L}$ ).
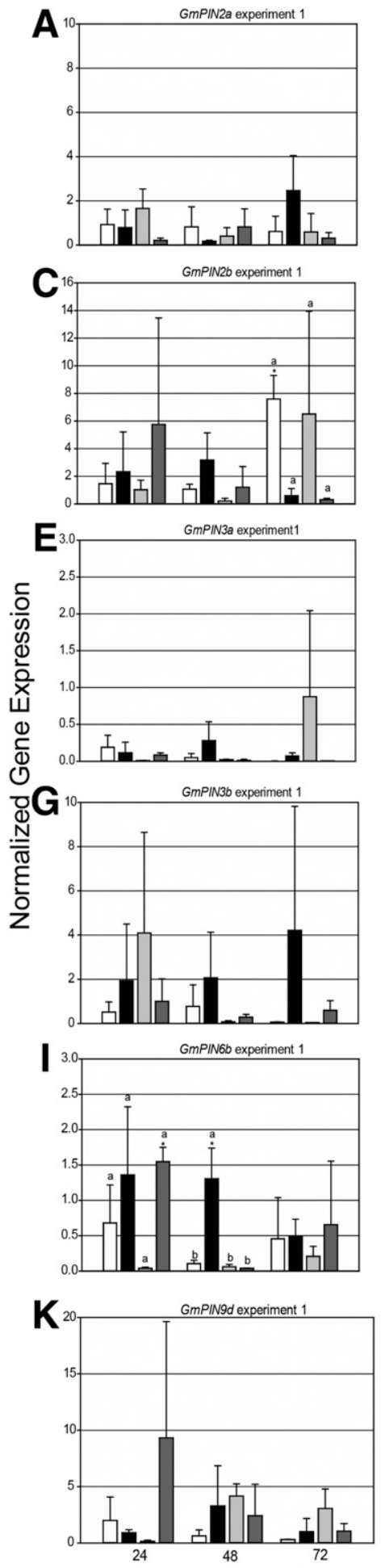

Hours post inoculation (hpi)

\section{DISCUSSION}

This study utilized two approaches to compare and investigate alterations in auxin levels in soybean roots during infection with $P$. sojae. Specifically, the work quantified both metabolites and the

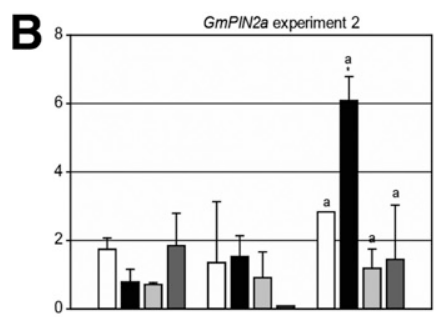

D

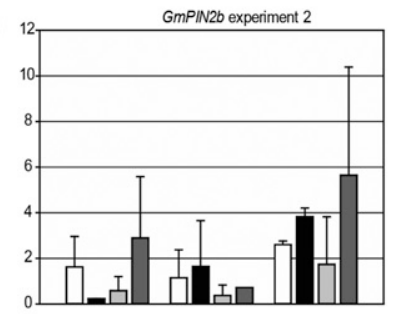

F

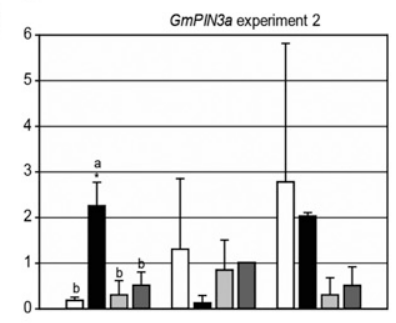

$\mathbf{H}$

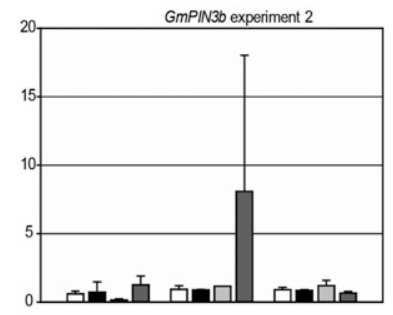

$\mathbf{J}$

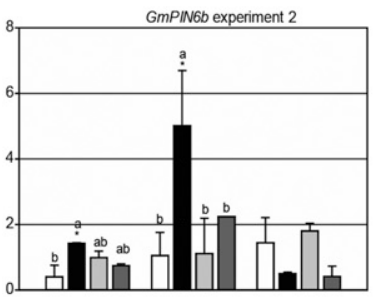

$\mathbf{L}$

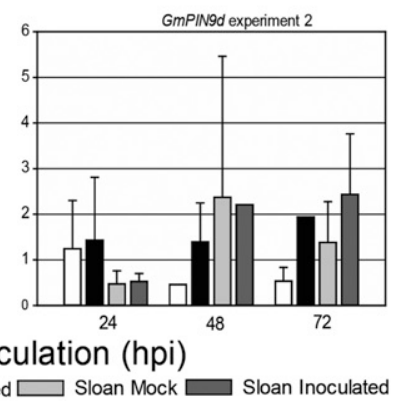

Fig. 8. Changes in gene expression for $\mathbf{A}$ and B, GmPIN2a (Glyma.13g101900), C and D, GmPIN2b (Glyma.17g057300), E and F, GmPIN3a (Glyma.07g217900), G and H, GmPIN3b (Glyma.20g014300), I and J, GmPIN6b (Glyma.14g120900), and K and L, GmPIN9d (Glyma.15g208600) relative to GmUBI3 (Chai et al. 2015; Wang et al. 2015) in Conrad and Sloan mock and inoculated roots in two independent experiments. Bars with different letters are significantly different from each other within a time point by Tukey's honest significant difference at $95 \%$ confidence. Asterisks indicate significant difference between mock and inoculated within cultivar at a given time point by Student's $t$ test (*, $P<0.05 ; * *, P<0.01$; and $* * *, P<0.001)$. Data represent means of two biological reps with three technical reps. Error bars represent standard deviation. 
expression of GmPIN transport genes in a moderately resistant and a moderately susceptible cultivar. To supplement these measurements, the levels of other hormones commonly involved in pathogen responses were also examined. Based on the results of several experiments, we showed that levels of an auxin precursor, Trp, were very high in mycelia and its presence in the medium at the concentrations measured suggests that it could be secreted into plants, where it has the potential to impact Trp-dependent auxin metabolism. We also showed that between 48 and $72 \mathrm{hpi}$, the level of auxin and related metabolites were higher in the inoculated versus mock roots of both cultivars. Furthermore, the increased expression of GmPIN genes suggests that auxin transport processes could be active during the infection response, modulating localized auxin concentration. Previous studies in our lab found that auxin-responsive genes had higher expression in the susceptible cultivar (Wang et al. $2012 \mathrm{~b}$ ), and that an $A U X / I A A$ repressor of auxin signaling was downregulated $24 \mathrm{~h}$ earlier in the susceptible cultivar than the resistant one (Wang et al. 2010; Zhou et al. 2009). Together these studies indicate that the cultivar with higher levels of quantitative disease resistance can modulate auxin levels, keeping them closer to those found under conditions of normal root growth and development, whereas in the susceptible cultivar, the presence of the pathogen results in increased levels of auxin.

Methods to directly detect and quantify auxin in specific tissues include thin-layer chromatography, high-pressure liquid chromatography, enzyme-linked immunosorbent assay, gas chromatography-mass spectrometry (GC-MS), and LC-MS/MS. In this study, LC-MS/MS methods were used for auxin quantification as they do not require the derivatization steps required in GC-MSbased auxin quantifications. They can also leverage the multiplereaction-monitoring function of triple quadrupole tandem mass spectrometers (QQQ LC-MS/MS) to quantify specific mass transitions for auxin and auxin metabolites, increasing the sensitivity of these methods logarithmically over GC-MS analyses (Blakeslee and Murphy 2016). This approach also allowed for the quantification of multiple hormones and related metabolites from a single sample. It has previously been used to simultaneously quantify a suite of hormones synthesized in response to pathogens and beneficial organisms, including IAA amino acid conjugates, SA and JA (Wang et al. 2017; Zhang et al. 2018).

Previous studies have observed colonization of soybean root tissue by $P$. sojae from 6 to 48 hpi (Enkerli et al. 1997; Qutob et al. 2000; Torto-Alalibo et al. 2007; Wang et al. 2011; Xiong et al. 2014; Yan et al. 2019). Therefore, the pathogen was likely present in the tissues analyzed in the current study, but probably not at the same level of colonization in each root sample across all experiments. Because of this, it is not possible to determine whether the Trp detected in root samples were derived from the plant or the pathogen, particularly since our data indicate that $P$. sojae is capable of releasing Trp into its surroundings, either through transport or as a result of tissue damage. While Trp is an essential amino acid synthesized by both the host and the pathogen for protein synthesis, recent work on the sunflower downy mildew pathogen Plasmopara halstedii suggests that intermediates of the brassinosteroid biosynthesis pathway could be imported from the host, converted to brassinolide, and then used to promote virulence (Sharma et al. 2015). Therefore, the possibly that oomycete pathogens use make use of both host as well as their own metabolic pathways to generate a specific metabolite cannot be ruled out and may not be unprecedented (Judelson 2017). In other words, it is possible that Trp secreted by $P$. sojae may impact Trp-dependent auxin synthesis in host plants. To fully determine the source of Trp detected in inoculated roots in our study, it would be necessary to feed either the plant or the pathogen an isotopically labeled Trp and then trace the isotope through the synthesis pathway using a time course LC-MS/ MS-based study. Even though our inoculations with $P$. sojae zoospores were successful, the number that successfully invaded roots and the rate of colonization of host cells is unknown. Thus, differences in the overall amount and stage of colonization may have contributed to the overall variability of the study.

The main goal of measuring Trp levels in our experiments was to quantify the levels of this compound as a potential auxin precursor. Since Trp-dependent synthesis may be responsible for producing the bulk of auxin in soybean, we hypothesized that higher levels of Trp, either synthesized endogenously or fed into the root by the pathogen, would correspond to higher levels of IAA. While its total concentrations were much higher than the hormone levels, especially in soybean roots, this was expected, given that approximately $20 \%$ of the carbon in plants is routed into aromatic amino acid synthesis (Coruzzi and Last 2000), while hormone levels in plant tissues are very low compared with primary metabolites, such as amino acids, sugars, or membrane lipids (Glauser et al. 2014). It should be noted, however, that the levels of hormones reported in the current study were comparable, in terms of plant mass (nanogram per gram), to those previously reported in other studies (Staswick et al. 2005; Yin et al. 2014; Zhang et al. 2016, 2018).

In the inoculated root assays, Trp, IAA, and IAA-conjugate levels increased in Sloan inoculated roots compared with the mock control in both experiments, although for Trp and IAA there was a $24 \mathrm{~h}$ shift from experiment one to experiment two. This was likely due to slight shifts in the level of infection or plant developmental stage between the two studies, as well as to differences in pathogen penetration and spread mentioned above. Glyma.08g312300, a gene involved in Trp biosynthesis, was upregulated in inoculated roots compared with the mock control of both Conrad and Sloan at 72 and 120 hpi in previous microarray studies (Wang et al. 2010; Zhou et al. 2009). Another study utilizing an untargeted metabolomics approach reported that Trp and other amino acid levels increased at 48 hpi in soybean hypocotyls inoculated with Rhizoctonia solani (Aliferis et al. 2014). Additionally, Yin et al. (2014) found higher levels of IAA in wheat leaves inoculation with Puccinia graminis f. sp. tritici at the later stages of infection, and Brauer et al. (2019) reported higher levels of IAA in a susceptible wheat cultivar inoculated with $F$. graminearum compared with two resistant cultivars. IAA-Glu levels also increased in inoculated roots compared with mock controls within each cultivar at $72 \mathrm{hpi}$, which suggests that IAA catabolism may be upregulated in both cultivars in an attempt to maintain root auxin homeostasis during infection. The higher levels of IAA-Ala in Sloan inoculated roots at 72 hpi further suggests that Sloan needs to activate more catabolic pathways and may be less capable of regulating auxin levels through other processes such as transport compared with Conrad.

The higher levels of IAA, IAA-Glu, and IAA-Ala detected in Sloan inoculated roots also correlate well with increased expression of Glyma.19g161000 and Glyma.19g221900, an auxin-induced gene and an auxin-responsive transcription factor (Wang et al. 2012b). Interestingly, an Aux/IAA signaling gene, Glyma. $19 \mathrm{~g} 181900$, was down-regulated in inoculated Sloan roots beginning at $48 \mathrm{hpi}$ and in inoculated Conrad roots beginning at 72 hpi (Wang et al. 2010; Zhou et al. 2009). In the absence of IAA, Aux/IAA proteins suppress auxin signaling by binding to a subset of auxin-responsive transcription factors (reviewed in RobertSeilaniantz et al. 2011; Weijers and Wagner 2016; Woodward and Bartel 2005). Activation of auxin signaling is known to enhance disease development in other pathosystems (Kidd et al. 2011; Liu et al. 2016; Navarro et al. 2006), and auxin-responsive genes had higher levels of expression in a susceptible wheat cultivar compared with resistant cultivars during infection with $F$. graminearum (Brauer et al. 2019). Therefore, we speculate that $P$. sojae could be targeting this gene in order to allow continued activation of auxin signal/responses to promote disease and that Conrad is able to delay this activation compared with Sloan.

Aside from catabolism, transport could also be used to remove IAA from plant cells. Therefore, to begin to investigate this, the expression of the GmPIN efflux genes was examined. GmPINIa 
(Glyma.08g054700) at 24 hpi, GmPIN6b (Glyma.14g120900) at 48 hpi, and GmPIN1b (Glyma.07g102500), GmPINle (Glyma. $19 \mathrm{~g} 128800$ ), and GmPIN2a (Glyma.13g101900) at $72 \mathrm{hpi}$ had higher expression in Conrad inoculated roots compared with mock controls. Two additional genes, GmPIN2b (Glyma.17g057300) and GmPIN9d (Glyma.15g208600) had similar, but nonsignificant, trends at 48 hpi. Similar trends were observed in Sloan inoculated roots only for GmPIN $1 b$ at 24 and $72 \mathrm{hpi}$ and for GmPIN2b at $24 \mathrm{hpi}$. The remaining four genes had no clear trends in expression level in either experiment. This suggests that the moderately resistant Conrad is more likely to activate the expression of GmPINs during infection with $P$. sojae than the moderately susceptible Sloan, which might lead to an increase in auxin efflux in the root. It also suggests that different GmPIN proteins may have different roles during the infection process. Differing roles of PIN family members have also been reported in Arabidopsis during infection with the cyst nematode, Heterodera schachtii, (Grunewald et al. 2009) and the root knot nematode, Meloidogyne incognita (Kyndt et al. 2016). However, whether or not the increased gene expression observed in the current study actually results differences in auxin transport would need to be confirmed with protein localization and auxin deposition assays, as the activity of PINs is also controlled by several posttranslational processes (reviewed in Titapiwatanakun and Murphy 2009).

The expression results reported here are in contrast to a previous study, where GmPINle was up-regulated in Sloan at 72 and $120 \mathrm{hpi}$ but down-regulated in Conrad (Wang et al. 2010). In the previous study, a tray test that involved wounding the plants and covering the wound with a mycelial slurry was used. As auxin is also involved in the wound response (reviewed in León et al. 2001), the inoculation method used in previous studies may have contributed to observed changes in GmPINle expression. To avoid this potential issue, we used a wound-free, zoospore-based inoculation method in this study.

Our metabolomic and expression data suggest that susceptible and resistant soybean cultivars engage different auxin responses following pathogen attack. An increasing body of work indicates that plant-microbe interactions are dependent upon localized auxin concentrations, which are regulated by the aggregate balance of synthesis, local and long-distance transport, and catabolism, and that regulation of IAA transport streams play a critical role in pathogen resistance. For example, a study examining the soybeanHeterodera glycines interaction found that an AtPIN3 homolog was up-regulated in syncytia in Williams 82 at $2 \mathrm{dpi}$, but that auxin signaling genes were down-regulated compared with uninfected control (Ithal et al. 2006). In contrast, an RNAseq analysis found that auxin signaling and transport genes, including the AtPIN1 homolog SlsoPIN1b, were overrepresented in genes down-regulated at $48 \mathrm{hpi}$ in a tomato genotype resistant to Ralstonia solanacearum but not in the susceptible genotype (French et al. 2018). Additionally, the auxin transport mutant diageotropica 1-1 (dgt1-1) was more resistant to $R$. solanacearum compared with the wild type (French et al. 2018). Zhao et al. (2019) found that auxin metabolism genes, auxin signaling genes, and auxin transporters including AtPIN4, AtPIN5, and AtPIN6 were up-regulated in $R$. solanacearum inoculated Arabidopsis roots at 12 to 48, 48, and 72 to $96 \mathrm{hpi}$, respectively. Transcriptional and metabolomic analyses of three different wheat cultivars inoculated with $F$. graminearum have shown that the susceptible genotype accumulated higher amounts of auxin and had the highest expression of auxin-related genes (Brauer et al. 2019). Transgenic A. thaliana expressing the effector PSE1 likely had altered auxin efflux and was more susceptible to $P$. parasitica (Evangelisti et al. 2013). Our data add to this body of work and support a model in which microbes attempt to alter homeostasis by increasing synthesis of IAA. Moderately resistant plants are able to successfully counter this disruption by engaging transport machinery and dissipating local increases in IAA. The more susceptible cultivar appears to be unable to utilize this mechanism and instead attempts to partially recover steady-state auxin levels by increasing catalysis of auxin.

Interestingly, but not surprisingly, IAA was not the only phytohormone significantly disrupted by $P$. sojae infection; JA levels tended to increase in inoculated roots compared with mock controls for both cultivars at 48 and 72 hpi. Previous microarray studies also found that Glyma.02g099200, an allene oxide cyclase gene in the JA synthesis pathway, was up-regulated in inoculated versus mock tissue in Conrad and Sloan at 48 and $72 \mathrm{hpi}$, in Conrad inoculated versus mock tissue at $120 \mathrm{hpi}$, and in Conrad inoculated versus Sloan inoculated tissue at $120 \mathrm{hpi}$ (Wang et al. 2010; Zhou et al. 2009). Traditionally, SA is associated with resistance to biotrophs and hemibiotrophs, while JA, along with ethylene (ET), is associated with resistance to necrotrophs (Pieterse et al. 2012; Vlot et al. 2009). In the soybean-P. sojae system, SA has been shown to be important in defense responses in hypocotyl and leaf tissue (Jing et al. 2015; Shine et al. 2016). Shine et al. (2016) found that SA levels were significantly higher in leaf tissue of the cultivars Harosoy and Harosoy 63 at 48 hpi with $P$. sojae. When the soybean SA biosynthesis genes Phenylalanine Ammonia Lyase (GmPAL) and Isochorismate Synthase (GmICS) where silenced in Harosoy, the percentage of surviving plants was lower at 6 and 8 dpi compared with the empty vector control. However, the percent survival of Harosoy 63 and Williams 82, which contain Response to Phytophthora sojae la (Rpsla) and Rpslk, respectively, was unaffected when these cultivars were inoculated with a race to which they are resistant (Shine et al. 2016). Jing et al. (2015) found that Phytoalexin Deficient 4 (PAD4) and Salicylic Acid Deficient 2 (SID2) were up-regulated in Williams 82 when inoculated with an avirulent race of $P$. sojae but were down-regulated when inoculated with a virulent race. The SA responsive genes, PathogenesisRelated 1 (PRI) and PR10 were up-regulated in both cases but to a greater degree in plants inoculated with the virulent isolate (Jing et al. 2015). Yan et al. (2019) also found that SA-related genes were down-regulated at 24 and 36 hpi in hairy roots when Defender Against Cell Death 1 (GmDAD1) was silenced compared with empty vector controls. These roots were also more susceptible to $P$. sojae (Yan et al. 2019). While some synergistic interaction between SA and JA does occur, the two hormones are also known to be antagonistic to each other (Glazebrook 2005; Pieterse et al. 2012; Wang et al. 2017). Yan et al. (2019) also reported that GmDAD1 silenced hairy roots had higher expression of the JA-responsive gene, Plant Defensin 1.2 (PDF1.2), at 24 and 36 hpi. However, Jing et al. (2015) found that the JA signaling suppressor, Jasmonate-ZimDomain 1 (JAZ1), had lower expression and the JA-responsive gene, $M Y C 3$, had higher expression in Williams 82 inoculated with an avirulent isolate at 12 to 36 hpi compared with plants inoculated with a virulent isolate, indicating that the JA response was active in $R p s$-mediated defense in etiolated hypocotyls (Jing et al. 2015). Our results suggest that while both SA and JA seem to increase in inoculated roots, JA seems to be particularly important at 48 and 72 hpi in Conrad and Sloan, which do not have Rps genes and are dependent on quantitative resistance. Yan et al. (2019) did not examine the expression of SA- and JA-responsive genes beyond 36 hpi and did not report the cultivar used to generate hairy roots. During this 48 to 72 hpi time frame, $P$. sojae is transitioning from biotrophy to necrotrophy (Beagle-Ristaino and Rissler 1983; Enkerli et al. 1997), which is marked by a turnover in expression of necrosis-preventing effector genes to necrosis-inducing effector genes (Qutob et al. 2000; Torto-Alalibo et al. 2007). Therefore, in roots SA-mediated defenses maybe more important during the early-biotrophic stages of infection, while JA-mediated defenses are more effective as $P$. sojae transitions to necrotrophy.

The overall outcome of resistance or susceptibility in a given pathosystem is regulated by a delicate balance of hormone crosstalk that is influenced by host genotype (Glazebrook 2005; Häffner et al. 2014). Häffner et al. (2014) found that an Arabidopsis ecotype with higher resistance to systemic colonization to Verticillium 
longisporum had higher levels of SA than an ecotype that was more resistant to stunting induced by the same pathogen. However, the stunting-resistant ecotype had higher levels of JA (Häffner et al. 2014). In a study examining the role of SA, JA, and ET pathways in the quantitative resistance of four potato cultivars to $P$. infestans, only one of the two varieties with higher quantitative resistance had an increase in SA during infection (Saubeau et al. 2016). One resistant wheat cultivar had significantly higher levels of SA than the susceptible control after inoculation with $F$. graminearum, while another resistant cultivar had significantly lower levels of both SA and JA (Brauer et al. 2019).

Furthermore, there is also crosstalk between hormones. Treatment with the SA analog benzothiadiazole S-methylester resulted in downregulation of IAA-responsive genes, such as Small Auxin Up Regulated (SAUR) and Aux/IAA family members (Wang et al. 2007), and SA activates a GH3 gene, WESO1 (Wes1), in Arabidopsis (Park et al. 2007). Transgenic Arabidopsis plants that constitutively expressed Wesl also had higher expression of PRI (Park et al. 2007). Additionally, a $0.5 \mathrm{mM}$ SA treatment prevented expression of the auxin activated DR5:: $\beta$-glucuronidase (GUS) reporter, even when the synthetic auxin 1-naphthalacetic acid (NAA) was also present, indicating that SA generally represses auxin signaling/responses (Wang et al. 2007). Expression of PR1 was higher in Arabidopsis plants inoculated with mutants of Pseudomonas syringae DC3000 with impaired IAA synthesis compared with those infected with the wild-type strain (McClerklin et al. 2018). In contrast, methyl jasmonate (MeJA) activates auxin biosynthesis in Arabidopsis by triggering the expression of IAA biosynthesis genes (Sun et al. 2009). Interestingly, these same genes are also up-regulated during infection with the necrotroph Alternaria brassicicola (Qi et al. 2012). Treating Arabidopsis with both MeJA and IAA had a synergistic effect on the expression of $P D F 1.2$. Thus, it appears that MeJA and IAA act synergistically during infection with necrotrophic pathogens (Qi et al. 2012). If a similar crosstalk among SA, JA, and IAA occurs in soybean, this would have important implications on infection with $P$. sojae. The timing of the activation of these pathways could be critical as they could lead to host resistance or susceptibility depending on the stage of infection and the relative concentrations of the hormones involved. More work investigating the downstream responses to these hormones is needed to determine, what, if any affect these pathways, both individually and together, have on the progression of the disease.

In summary, levels of IAA and its metabolites increase in soybean roots at 48 and 72 hpi with $P$. sojae, especially in the moderately susceptible cultivar Sloan, and five GmPIN genes had higher expression in the moderately resistant cultivar Conrad during a time course assay in at least one of the three time points examined. In combination with our previous expression data (Wang et al. 2010, 2012b; Zhou et al. 2009), we conclude that IAA enhances susceptibility of soybean to $P$. sojae. IAA is known to trigger cellwall loosening (Ren and Gray 2015; Spartz et al. 2014) and production of reactive oxygen species (ROS) (Joo et al. 2001, 2005). Therefore, we speculate that $P$. sojae releases effectors and Trp into host roots, which enhances IAA biosynthesis, transport, and signaling/responses. This loosens cell walls, allowing formation of haustoria during the early, biotrophic stages of infection. As IAA levels continue to accumulate, ROS levels increase, which contributes to host cell death during the later, necrotrophic stage of the disease. If the increased expression of GmPINs observed in Conrad does indeed translate into increased auxin efflux, it is possible that this will allow a faster return to auxin homeostasis via export of IAA that mitigates some of the $P$. sojae-induced susceptibility. In contrast, Sloan activates additional IAA catabolism pathways, which appear to be less effective at reducing susceptibility. Future work should examine the expression and activity of additional IAA signaling/response genes/proteins and the accumulation of auxin transport proteins. The latter will be greatly aided by several recent studies that have identified soybean homologs of the main groups of Arabidopsis IAA transporters (Chai et al. 2016; Liu and Wei 2017; Wang et al. 2015). This information could be used to generate transgenic soybean with knockouts in one or more GmPINs or could be used to select recently released fast neutron mutants (Bolon et al. 2011) with insertions or deletions in auxin-related genes. These plants could then be screened for increased or decreased susceptibility to identify candidate genes and molecular markers for developing cultivars with high levels of quantitative disease resistance toward $P$. sojae.

\section{ACKNOWLEDGMENTS}

We thank Deloris J. Veney and Yiyun Lin for technical assistance and David Mackey and William Rolling for critical review of the manuscript.

\section{LITERATURE CITED}

Aliferis, K. A., Faubert, D., and Jabaji, S. 2014. A metabolic profiling strategy for the dissection of plant defense against fungal pathogens. PLoS One 9: e111930.

Beagle-Ristaino, J. E., and Rissler, J. F. 1983. Histopathology of susceptible and resistant soybean roots inoculated with zoospores of Phytophthora megasperma f. sp. glycinea. Phytopathology 73:590-595.

Benjamins, R., and Scheres, B. 2008. Auxin: The looping star in plant development. Annu. Rev. Plant Biol. 59:443-465.

Berens, M. L., Berry, H. M., Mine, A., Argueso, C. T., and Tsuda, K. 2017. Evolution of hormone signaling networks in plant defense. Annu. Rev. Phytopathol. 55:401-425.

Blakeslee, J. J., and Murphy, A. S. 2016. Microscopic and biochemical visualization of auxins in plant tissues. Pages 37-53 in: Environmental Responses in Plants: Methods and Protocols. Methods in Molecular Biology, vol. 1398. P. Duque, ed.Humana Press, New York.

Blakeslee, J. J., Spatola Rossi, T., and Kriechbaumer, V. 2019. Auxin biosynthesis: Spatial regulation and adaptation to stress. J. Exp. Bot. 70:5041-5049.

Boivin, S., Fonuni-Farde, C., and Frugier, F. 2016. How auxin and cytokinin phytohormones modulate roots microbe interactions. Front. Plant Sci. 7:1240.

Bolon, Y.-T., Huan, W. J., Xu, W. W., Grant, D., Stacey, M. G., Nelson, R. T., Gerhardt, D. J., Jeddeloh, J. A., Stacey, G., Muehlbauer, G. J., Orf, J. H., Naeve, S. L., Stupar, R. M., and Vance, C. P. 2011. Phenotypic and genomic analyses of a fast neutron mutant population resource in soybean. Plant Physiol. 156:240-253.

Brauer, E. K., Rocheleau, H., Balcerzak, M., Pan, Y., Fauteux, F., Liu, Z., Wang, L., Zheng, W., and Ouellet, T. 2019. Transcriptional and hormonal profiling of Fusarium graminearum-infected wheat reveals an association between auxin and susceptibility. Physiol. Mol. Plant Pathol. 107:33-39.

Chai, C., Wang, Y., Joshi, T., Valliyodan, B., Prince, S., Michel, L., Xu, D., and Nguyen, H. T. 2015. Soybean transcription factor ORFeome associated with drought resistance: A valuable resource to accelerate research on abiotic stress resistance. BMC Genomics 16:596.

Chai, C., Wang, Y., Valliyodan, B., and Nguyen, H. T. 2016. Comprehensive analysis of the soybean (Glycine max) GmLAX auxin transporter gene family. Front. Plant Sci. 7:282.

Chilton, M.-D., Drummond, M. H., Merlo, D. J., Sciaky, D., Montoya, A. L., Gordon, M. P., and Nester, E. W. 1977. Stable incorporation of plasmid DNA into higher plant cells: The molecular basis of crown gall tumorigenesis. Cell 11:263-271.

Contreras-Cornejo, H. A., López-Bucio, J. S., Méndez-Bravo, A., Macías-Rodríguez, L., Ramos-Vega, M., Guevara-García, Á. A., and López-Bucio, J. 2015. Mitogen-activated protein kinase 6 and ethylene and auxin signaling pathways are involved in Arabidopsis root-system architecture alterations by Trichoderma atroviride. Mol. Plant-Microbe Interact. 28:701-710.

Contreras-Cornejo, H. A., Macías-Rodríguez, L., Cortés-Penagos, C., and López-Bucio, J. 2009. Trichoderma virens, a plant beneficial fungus, enhances biomass production and promotes lateral root growth through an auxin-dependent mechanism in Arabidopsis. Plant Physiol. 149:1579-1592.

Coruzzi, G., and Last, R. 2000. Amino acids. Pages 358-410 in: Biochemistry and Molecular Biology of Plants. B. Buchanan, W. Gruissem, and R. Jones, eds. American Society of Plant Biologists, Maryland.

Domingo, C., Andrés, F., Tharreau, D., Iglesias, D. J., and Talón, M. 2009. Constitutive expression of OsGH3.1 reduces auxin content and enhances defense response and resistance to a fungal pathogen in rice. Mol. PlantMicrobe Interact. 22:201-210.

Enkerli, K., Hahn, M. G., and Mims, C. W. 1997. Ultrastructure of compatible and incompatible interactions of soybean roots infected with the plant pathogenic oomycete Phytophthora sojae. Can. J. Bot. 75:1493-1508. 
Evangelisti, E., Govetto, B., Minet-Kebdani, N., Kuhn, M., Attard, A., Ponchet, M., Panabières, F., and Gourgues, M. 2013. The Phytophthora parasitica RXLR effector penetration-specific effector 1 favors Arabidopsis thaliana infection by interfering with auxin physiology. New Phytol. 199: 476-489.

French, E., Kim, B.-S., Rivera-Zuluaga, K., and Iyer-Pascuzzi, A. A. 2018. Whole root transcriptomic analysis suggests a role for auxin pathways in resistance to Ralstonia solanacearum. Mol. Plant-Microbe Interact. 31:432-444.

Friml, J., Benková, E., Blilou, I., Winiewska, J., Hamann, F., Ljung, K., Woody, S., Sandberg, G., Scheres, B., Jürgens, G., and Palme, K. 2002a. AtPIN4 mediates sink-driven auxin gradients and root patterning in Arabidopsis. Cell 108:661-673.

Friml, J., Winśniewska, J., Benková, E., Mendgen, K., and Palme, K. 2002 b. Lateral relocation of auxin efflux regulator PIN3 mediates tropism in Arabidopsis. Nature 415:806-809.

Gälweiler, L., Guan, C., Müller, A., Wisman, E., Mendgen, K., Yephremov, S., and Palme, K. 1998. Regulation of polar auxin transport by AtPIN1 in Arabidopsis vascular tissue. Science 282:2226-2230.

Gao, S., Fang, J., Xu, F., Wang, W., Sun, X., Chu, J., Cai, B., Feng, Y., and Chu, C. 2014. Cytokinin oxidase/dehydrogenase4 integrates cytokinin and auxin signaling to control rice crown root formation. Plant Physiol. 165: 1035-1046.

Glauser, G., Vallat, A., and Balmer, D. 2014. Hormone profiling. Pages 597-608 in: Arabidopsis Protocols Methods in Molecular Biology, vol. 1062. J. Sanchez-Serrano and J. Salinas, eds. Humana Press, New York.

Glazebrook, J. 2005. Contrasting mechanisms of defense against biotrophic and necrotrophic pathogens. Annu. Rev. Phytopathol. 43:205-227.

Glickmann, E., Gardan, L., Jacquet, S., Hussain, S., Elasri, M., Petit, A., and Dessaux, Y. 1998. Auxin production is a common feature of most pathovars of Pseudomonas syringae. Mol. Plant-Microbe Interact. 11:156-162.

Govindarajulu, M., Elmore, J. M., Fester, T., and Taylor, C. G. 2008. Evaluation of constitutive viral promoters in transgenic soybean roots and nodules. Mol. Plant-Microbe Interact. 21:1027-1035.

Govindarajulu, M., Kim, S.-Y., Libault, M., Berg, R. H., Tanaka, K., Stacey, G., and Taylor, C. G. 2009. GS52 ecto-apyrase plays a critical role during soybean nodulation. Plant Physiol. 149:994-1004.

Grant, D., Nelson, R. T., Cannon, S. B., and Shoemaker, R. C. 2010. SoyBase, the USDA-ARS soybean genetics and genomics database. Nucleic Acids Res. 38:D843-D846.

Grunewald, W., Cannoot, B., Friml, J., and Gheysen, G. 2009. Parasitic nematodes modulate PIN-mediated auxin transport to facilitate infection. PLoS Pathog 5:e1000266.

Häffner, E., Karlovsky, P., Splivallo, R., Traczewska, A., and Diederichsen, E. 2014. ERECTA, salicylic acid, abscisic acid, and jasmonic acid modulate quantitative disease resistance of Arabidopsis thaliana to Verticillium longisporum. BMC Plant Biol. 14:85.

Hoitink, H. A. J., and Schmitthenner, A. F. 1969. Rhododendron wilt caused by Phytophthora citricola. Phytopathology 59:708-709.

Ithal, N., Recknor, J., Nettleson, D., Maier, T., Baum, T. J., and Mitchem, M. G. 2006. Developmental transcript profiling of cyst nematode feeding cells in soybean roots. Mol. Plant-Microbe Interact. 20:510-525.

Jing, M., Ma, H., Li, H., Guo, B., Zhang, X., Ye, W., Wang, H., Wang, Q., and Wang, Y. 2015. Differential regulation of defense-related proteins in soybean during compatible and incompatible interactions between Phytophthora sojae and soybean by comparative proteomic analysis. Plant Cell Rep. 34:1263-1280

Joo, J. H., Bae, Y. S., and Lee, J. S. 2001. Role of auxin-induced reactive oxygen species in root gravitropism. Plant Physiol. 126:1055-1060.

Joo, J. H., Yoo, H. J., Hwang, I., Lee, J. S., Nam, K. H., and Bae, Y. S. 2005. Auxin-induced reactive oxygen species production requires the activation of phosphatidylinositol 3-kinase. FEBS Lett. 579:1243-1248.

Judelson, H. 2017. Metabolic diversity and novelties in the oomycetes. Annu. Rev. Microbiol. 71:21-39.

Kidd, B. N., Kadoo, N. Y., Dombrecht, B., Tekeoğlu, M., Gardiner, D. M., Thatcher, L. F., Aitken, E. A. B., Schenk, P. M., Manners, J. M., and Kazan, K. 2011. Auxin signaling and transport promote susceptibility to the rootinfecting fungal pathogen Fusarium oxysporum in Arabidopsis. Mol. PlantMicrobe Interact. 24:733-748.

Kohlen, W., Ng, J. L. P., Deinum, E. E., and Mathesius, U. 2018. Auxin transport, metabolism, and signalling during nodule initiation: Indeterminate and determinate nodules. J. Exp. Bot. 69:229-244.

Korasick, D. A., Enders, T. A., and Strader, L. C. 2013. Auxin biosynthesis and storage forms. J. Exp. Bot. 64:2541-2555.

Kunkel, B. N., and Harper, C. P. 2018. The roles of auxin during interactions between bacterial plant pathogens and their hosts. J. Exp. Bot. 69:245-254.

Kyndt, T., Goverse, A., Haegeman, A., Warmerdam, S., Wanjau, C., Jahani, M., Engler, G., de Almeida Engler, J., and Gheysen, G. 2016. Redirection of auxin flow in Arabidopsis thaliana roots after infection by root-knot nematodes. J. Exp. Bot. 67:4559-4570.
León, J., Rojo, E., and Sánchez-Serrano, J. J. 2001. Wound signaling in plants. J. Exp. Bot. 52:1-9.

Libault, M., Thibivilliers, S., Bilgin, D. D., Radwan, O., Benitez, M., Clough, S. J., and Stacey, G. 2008. Identification of four soybean reference genes for gene expression normalization. Plant Gene 1:44-54.

Liu, Y., Guo, Y., Ma, C., Zhang, D., Wang, C., and Yang, Q. 2016. Transcriptome analysis of maize resistance to Fusarium graminearum. BMC Genomics 17:477.

Liu, Y., and Wei, H. 2017. Genome-wide identification and evolution of the PIN-FORMED (PIN) gene family in Glycine max. Genome 60:564-571.

Ludwig-Müller, J. 2011. Auxin conjugates: Their role for plant development and in the evolution of land plants. J. Exp. Bot. 62:1757-1773.

Ludwig-Müller, J. 2015. Bacteria and fungi controlling plant growth by manipulating auxin: Balance between development and defense. J. Plant Physiol. 172:4-12.

Luschnig, C., Gaxiola, R. A., Grisafi, P., and Fink, G. R. 1998. EIR1, a rootspecific protein involved in auxin transport, is required for gravitropism in Arabidopsis thaliana. Genes Dev. 12:2175-2187.

Ma, K. W., and Ma, W. 2016. Phytohormone pathways as targets of pathogens to facilitate infection. Plant Mol. Biol. 91:713-725.

Mashiguchi, K., Tanaka, K., Sakai, T., Sugawara, S., Kawaide, H., Natsume, M., Hanada, A., Yaeno, T., Shirasu, K., Yao, H., McSteen, P., Zhao, Y., Hayashi, K., Kamiya, Y., and Kasahara, H. 2011. The main auxin biosynthesis pathway in Arabidopsis. Proc. Natl. Acad. Sci. 108:18512-18517.

McClerklin, S. A., Lee, S. G., Harper, C. P., Nwumeh, R., Jez, J. M., and Kunkel, B. N. 2018. Indole-3-acetaldehyde dehydrogenase-dependent auxin synthesis contributes to virulence of Pseudomonas syringae strain DC3000. PLoS Pathog 14:e1006811.

Mideros, S., Nita, M., and Dorrance, A. E. 2007. Characterization of components of partial resistance, Rps2, and root resistance to Phytophthora sojae in soybean. Phytopathology 97:655-662.

Müller, A., Guan, C., Gälweiler, L., Tänzler, P., Huijer, P., Marchant, A., Parry, G., Bennett, M., Wisman, E., and Palme, K. 1998. AtPIN2 defines a locus of Arabidopsis for root gravitropism control. EMBO J. 17:6903-6911.

Navarro, L., Dunoyer, P., Jay, F., Arnold, B., Dharmasiri, N., Estelle, M., Voinnet, O., and Jones, J. D. G. 2006. A plant miRNA contributes to antibacterial resistance by repressing auxin signaling. Science 312:436-439.

Ouyang, J., Shao, X., and Li, J. 2000. Indole-3-glycerol phosphate, a branchpoint of indole-3-acetic acid biosynthesis from the tryptophan biosynthetic pathway in Arabidopsis thaliana. Plant J. 24:327-333.

Park, J.-E., Park, J.-Y., Kim, Y.-S., Staswick, P. E., Jeon, J., Yun, J., Kim, S.-Y., Kim, J., Lee, Y.-H., and Park, C.-M. 2007. GH3-mediated auxin homeostasis links growth regulation with stress adaptation response in Arabidopsis. J. Biol. Chem. 282:10036-10046.

Peer, W. A., Blakeslee, J. J., Yang, H., and Murphy, A. S. 2011. Seven things we think we know about auxin transport. Mol. Plant 4:487-504.

Perley, J. E., and Stowe, B. B. 1966. On the ability of Taphrina deformans to produce indoleacetic acid from tryptophan by way of tryptamine. Plant Physiol. 41:234-237.

Pieterse, C. M. J., Van der Does, D., Zamioudis, C., Leon-Reyes, A., and Van Wees, S. C. M. 2012. Hormonal modulation of plant immunity. Annu. Rev. Phytopathol. 28:489-521.

Pieterse, C. M. J., Zamioudis, C., Berendsen, R. L., Weller, D. M., Van Wees, S. C. M., and Bakker, P. A. H. M. 2014. Induced systemic resistance by beneficial microbes. Annu. Rev. Phytopathol. 52:347-375.

Plett, J. M., Kemppainen, M., Kale, S. D., Kohler, A., Legué, V., Brun, A., Tyler, B. M., Pardo, A. G., and Martin, F. 2011. A secreted effector protein of Laccaria bicolor is required for symbiosis development. Curr. Biol. 21: 1197-1203.

Qi, L., Yan, J., Li, Y., Jiang, H., Sun, J., Chen, Q., Li, H., Chu, J., Yan, C., Sun, X., Yu, Y., Li, C., and Li, C. 2012. Arabidopsis thaliana plants differentially modulate auxin biosynthesis and transport during defense responses to the necrotrophic pathogen Alternaria brassicicola. New Phytol. 195:872-882.

Qutob, D., Hraber, P. T., Sobral, B. W. S., and Gijzen, M. 2000. Comparative analysis of expressed sequences in Phytophthora sojae. Plant Physiol. 123: 243-254

Ren, H., and Gray, W. M. 2015. SAUR proteins as effectors of hormonal and environmental signals in plant growth. Mol. Plant 8:1153-1164.

Rightmyer, A. P., and Long, S. R. 2011. Pseudonodule formation by wild type and symbiotic mutant Medicago truncatula in response to auxin transport inhibitors. Mol. Plant-Microbe Interact. 24:1372-1384.

Robert-Seilaniantz, A., Grant, M., and Jones, J. D. G. 2011. Hormone crosstalk in plant disease and defense: More than just jasmonate-salicylate antagonism. Annu. Rev. Phytopathol. 49:317-343.

Rosendahl, L., and Jochimsen, B. U. 1995. In vitro indole-3-acetic acid uptake in symbiosomes from soybean (Glycine max L.) root nodules. Symbiosis 19:99-110

Roy, S., Robson, F., Lilley, J., Liu, C.-W., Cheng, X., Wen, J., Walker, S., Sun, J., Cousins, D., Bone, C., Bennett, M. J., Downie, J. A., Swarup, R., 
Oldroyd, G., and Murray, J. D. 2017. MtLAX2, a functional homologue of the Arabidopsis auxin influx transporter AUX1, is required for nodule organogenesis. Plant Physiol. 174:326-338.

Saubeau, G., Perrin, F., Marnet, N., Andrivon, D., and Val, F. 2016. Hormone signaling pathways are differentially involved in quantitative resistance of potato to Phytophthora infestans. Plant Pathol. 65:342-352.

Sharma, R., Xia, X., Cano, L. M., Evangelisti, E., Kemen, E., Judelson, H., Oome, S., Sambles, C., van den Hoogen, D. J., Kitner, M., Klein, J., Meijer, H. J. G., Spring, O., Win, J., Zipper, R., Bode, H. B., Govers, F., Kamoun, S., Schornack, S., Studholme, D. J., Van den Ackerveken, G., and Thines, M. 2015. Genome analyses of the sunflower pathogen Plasmopara halstedii provide insights into effector evolution in downy mildews and Phytophthora. BMC Genomics 16:741.

Shen, C., Yue, R., Sung, T., Zhang, L., Xu, L., Tie, S., Wang, H., and Yang, Y. 2015. Genome-wide identification and expression analysis of Auxin response factor gene family in Medicago truncatula. Front. Plant Sci. 6:73.

Shine, M. B., Yang, J.-W., El-Habbak, M., Nagyabhyru, P., Fu, D.-Q., Navarre, D., Ghabrial, S., Kachroo, P., and Kachroo, A. 2016. Cooperative functioning between phenylalanine ammonia lyase and isochorismate synthase activities contributes to salicylic acid biosynthesis in soybean. New Phytol. 212:627-636.

Soeno, K., Goda, H., Ishii, T., Ogura, T., Tachikawa, T., Saski, E., Yoshida, S., Fujioka, S., Asami, T., and Shimada, Y. 2010. Auxin biosynthesis inhibitors, identified by a genomics-based approach, provides insights into auxin biosynthesis. Plant Cell Physiol. 51:524-536.

Spaepen, S., Vanderleyden, J., and Remans, R. 2007. Indole-3-acetic acid in microbial and microorganism-plant signaling. FEMS Microbiol. Rev. 31:425-448.

Spartz, A. K., Ren, H., Park, M. Y., Grandt, K. N., Lee, S. H., Murphy, A. S., Sussman, M. R., Overvoorde, P. J., and Gray, W. M. 2014. SAUR inhibition of PP2C-D phosphatase activates plasma membrane $\mathrm{H}^{+}$-ATPases to promote cell expansion in Arabidopsis. Plant Cell 26:2129-2142.

Stasko, A. K., Wickramasinghe, D., Nauth, B. J., Acharya, B., Ellis, M. L., Taylor, C. G., McHale, L. K., and Dorrance, A. E. 2016. High density mapping of resistance QTL towards Phytophthora sojae, Pythium irregulare, and Fusarium graminearum in the same soybean population. Crop Sci. 56:2476-2492.

Staswick, P. E., Serban, B., Rowe, M., Tiryaki, I., Maldonado, M. T., Maldonado, M. C., and Suza, W. 2005. Characterization of an Arabidopsis enzyme family that conjugates amino acids to indole-3-acetic acid. Plant Cell 17:616-627.

Sun, J., Xu, Y., Ye, S., Jiang, H., Chen, Q., Liu, F., Zhou, W., Chen, R., Li, X., Tietz, O., Wu, X., Cohen, J., Palme, K., and Li, C. 2009. Arabidopsis ASA1 is important for jasmonate-mediated regulation of auxin biosynthesis and transport during lateral root formation. Plant Cell 21:1495-1511.

Sziráki, I., Balázs, R., and Király, Z. 1975. Increased levels of cytokinin and indoleacetic acid in peach leaves infected with Taphrina deformans. Physiol. Plant Pathol. 5:45-50.

Thomashow, L. S., Reeves, S., and Thomashow, M. F. 1984. Crown gall oncogenesis: Evidence that a T-DNA gene from the Agrobacterium Ti plasmid pTiA6 encodes an enzyme that catalyzes synthesis of indoleacetic acid. Proc. Natl. Acad. Sci. 81:5071-5075.

Titapiwatanakun, B., and Murphy, A. S. 2009. Post-transcriptional regulation of auxin transport proteins: Cellular trafficking, protein phosphorylation, protein maturation, ubiquitination, and membrane composition. J. Exp. Bot. 60:1093-1107.

Tivendale, N. D., Ross, J. J., and Cohen, J. D. 2014. The shifting paradigms of auxin biosynthesis. Trends Plant Sci. 19:44-51.

Torto-Alalibo, T. A., Tripathy, S., Smith, B. M., Arredondo, F. D., Zhou, L., Li, H., Chibucos, M. C., Qutob, D., Gijzen, M., Mao, C., Sobral, B. W. S., Waugh, M. E., Mitchell, T. K., Dean, R. A., and Tyler, B. M. 2007. Expressed sequence tags from Phytophthora sojae reveals genes specific to development and infection. Mol. Plant-Microbe Interact. 20:781-793.

Turner, M., Nizampatnam, N. R., Baron, M., Cappin, S., Damodaran, S., Adihikari, S., Arunachdam, S. P., Yu, O., and Subramanian, S. 2013. Ectopic expression of miR160 results in auxin hypersensitivity, cytokinin hyposensitivity, and inhibition of symbiotic nodule development in soybean. Plant Physiol. 162:2042-2055.

Utsuno, K., Shikanai, T., Yamada, Y., and Hashimoto, T. 1998. AGR, an agravitropic locus of Arabidopsis thaliana, encodes a novel membraneprotein family member. Plant Cell Physiol. 39:1111-1118. van den Berg, T., Krover, R. A., Testerink, C., and ten Tusscher, K. H. W. J. 2016. Modeling halotropism: A key role for root tip architecture and reflux loop remodeling in redistributing auxin. Development 143:3350-3362.

Vlot, A. C., Dempsey, D. A., and Klessig, D. F. 2009. Salicylic acid, a multifaceted hormone to combat disease. Annu. Rev. Phytopathol. 47:177-206.

Wang, D., Pajerowska-Mukhtar, K., Hendrickson Culler, A., and Dong, X. 2007. Salicylic acid inhibits pathogen growth in plants through repression of the auxin signaling pathway. Curr. Biol. 17:1784-1790.

Wang, H., St. Martin, S. K., and Dorrance, A. E. 2012a. Comparison of phenotypic methods and yield contributions of quantitative trait loci for partial resistance to Phytophthora sojae in soybean. Crop Sci. 52:609-622.

Wang, H., Waller, L., Tripathy, S., St. Martin, S. K., Zhou, L., Krampis, K., Tucker, D. M., Mao, Y., Hoeschele, I., Maroof, M. A. S., Tyler, B. M., and Dorrance, A. E. 2010. Analysis of genes underlying soybean quantitative trait loci conferring partial resistance to Phytophthora sojae. Plant Gen. 3: 23-40.

Wang, H., Wijeratne, A., Wijeratne, S., Lee, S., Taylor, C. G., St. Martin, S. K., McHale, L. K., and Dorrance, A. E. 2012b. Dissection of two soybean QTL conferring partial resistance to Phytophthora sojae through sequence and gene expression analysis. BMC Genomics 13:428.

Wang, M., Rui, L., Yan, H., Shi, H., Zhao, W., Lin, J. E., Zhang, K., Blakeslee, J. J., Mackey, D., Tang, D., Wei, Z., and Wang, G.-L. 2017. The major leaf ferredoxin Fd2 regulates plant innate immunity in Arabidopsis. Mol. Plant Pathol. 19:1377-1390.

Wang, Q., Han, C., Ferreira, A. O., Yu, X., Ye, W., Tripathy, S., Kale, S. D., Gu, B., Sheng, Y., Sui, Y., Wang, X., Zhang, Z., Cheng, B., Dong, S., Shan, W., Zheng, X., Dou, D., Tyler, B. M., and Wang, Y. 2011. Transcriptional programming and functional interactions within the Phytophthora sojae RXLR effector repertoire. Plant Cell 23:2064-2086.

Wang, Y., Chai, C., Valliyodan, B., Maupin, C., Annen, B., and Nguyen, H. T. 2015. Genome-wide analysis and expression profiling of the PIN auxin transporter gene family in soybean (Glycine max). BMC Genomics 16:951.

Weijers, D., and Wagner, D. 2016. Transcriptional responses to the auxin hormone. Annu. Rev. Plant Biol. 67:539-574.

Woodward, A. W., and Bartel, B. 2005. Auxin: Regulation, action, and interaction. Ann. Bot. 95:707-735.

Xiong, Q., Ye, W., Choi, D., Wong, J., Qiao, Y., Tao, K., Wang, Y., and Ma, W. 2014. Phytophthora suppressor of RNA silencing 2 is a conserved RxLR effector that promotes infection in soybean and Arabidopsis thaliana. Mol. Plant-Microbe Interact. 27:1379-1389.

Yan, Q., Si, J., Cui, X., Peng, H., Jing, M., Chen, X., Xing, H., and Dou, D. 2019. GmDAD1, a conserved Defender against cell death 1 (DADI) from soybean, positively regulates plant resistance against Phytophthora pathogens. Front. Plant Sci. 10:107.

Yin, C., Park, J., Gang, D. R., and Hulbert, S. H. 2014. Characterization of a tryptophan 2-monooxygenase gene from Puccinia graminis f. sp. tritici involved in auxin biosynthesis and rust pathogenicity. Mol. Plant-Microbe Interact. 27:227-235

Zhang, J., Liu, J. E., Harris, C., Pareira, F. C. M., Wu, F., Blakeslee, J. J., and Peer, W. A. 2016. DAO1 catalyzes temporal and tissue-specific oxidative inactivation of auxin in Arabidopsis thaliana. Proc. Natl. Acad. Sci. 113: 11010-11015.

Zhang, Q., Berkey, R., Blakeslee, J. J., Lin, J., Ma, X., King, H., Liddle, A., Gus, L., Munnik, T., Wang, X., and Xiao, S. 2018. Arabidopsis phospholipase D $\alpha 1$ and D $\delta$ oppositely modulate EDS1- and SA-independent basal resistance against adapted powdery mildew. J. Exp. Bot. 69:3675-3688.

Zhao, C., Wang, H., Lu, Y., Hu, J., Qu, L., Li, Z., Wang, D., He, Y., Valls, M., Coll, N., Chen, Q., and Lu, H. 2019. Deep sequencing reveals early reprogramming of Arabidopsis root transcriptomes upon Ralstonia solanacearum infection. Mol. Plant-Microbe Interact. 32:813-827.

Zhao, Y., Christensen, S. K., Fankhauser, C., Cashman, J. R., Cohen, J. D., Weigel, D., and Chory, J. 2001. A role for flavin monooxygenase-like enzymes in auxin biosynthesis. Science 291:306-309.

Zhou, L., Mideros, S. X., Bao, L., Hanlon, R., Arredondo, F. D., Tripathy, S., Krampis, K., Jerauld, A., Evans, C., St. Martin, S. K., Maroof, M. A. S., Hoeschele, I., Dorrance, A. E., and Tyler, B. M. 2009. Infection and genotype remodel the entire soybean transcriptome. BMC Genomics 10:49. 